\title{
Modelo de riesgos competitivos para deserción y graduación en estudiantes universitarios de programas de pregrado de una universidad privada de Medellín (Colombia)
}

\author{
Bibiana A. Castro-Montoya ${ }^{1 *}$, Carlos M. Lopera-Gómez² , Rubén D. Manrique-Hernández ${ }^{3}$, y Difariney Gonzalez- \\ Gómez ${ }^{3,4}$. \\ (1) Grupo de investigación en Educación Superior, Universidad CES. Calle 10 a \# 22-04, Medellín-Colombia \\ (correo-e: bcastro@ces.edu.co; asegura@ces.edu.co) \\ (2) Grupo de investigación en Estadística Industrial, Universidad Nacional de Colombia, Medellín-Colombia. Cra. 65 \\ \#59a-110 (correo-e: cmlopera@unal.edu.co) \\ (3) Grupo de investigación en Epidemiología y Bioestadística, Universidad CES. Calle 10 a \# 22-04, Medellín-Colombia. \\ (correo-e: rmanrique@ces.edu.co; dgonzalez@ces.edu.co) \\ (4) Grupo de investigación en Demografía y Salud. Universidad de Antioquia. Calle 62 \#52-59. Medellín-Colombia \\ (correo-e: difariney.gonzalez@udea.edu.co)
}

* Autor a quien debe ser dirigida la correspondencia

Recibido May. 11, 2020; Aceptado Jul. 7, 2020; Versión final Ago. 3, 2020, Publicado Feb. 2021

\begin{abstract}
Resumen
El objetivo de esta investigación fue analizar factores demográficos, socioeconómicos y académicos asociados con deserción y graduación, mediante un modelo de riesgos competitivos. Se incluyeron 639 estudiantes matriculados en 2009-2010, a quienes se les realizó seguimiento durante 14 periodos académicos. Los resultados mostraron que la probabilidad acumulada de deserción para el segundo periodo académico fue de 0.147 . La probabilidad de graduarse en el tiempo estipulado por el programa fue del 0.187 y de 0.328 un año después. Para el periodo 14 , el $49.0 \%$ de los estudiantes había obtenido su título y el $35.4 \%$ habían desertado. Variables socioeconómicas estuvieron asociadas a la probabilidad de deserción, mientras que ciertas condiciones demográficas se asociaron con la probabilidad de graduación. No obstante, las variables académicas tuvieron un efecto significativo en ambos desenlaces. Se concluye que las características asociadas a la deserción y la graduación se corresponden con aspectos que se pueden intervenir por las instituciones educativas para incrementar la permanencia y graduación.
\end{abstract}

\section{A competing dropout and graduation risk survival analysis of undergraduate students at a private university in Medellín (Colombia)}

\begin{abstract}
The aim of this research study was to analyze demographic, socioeconomic, and academic factors associated with dropout and graduation using a competing risks model. A total of 639 undergraduate students enrolled in 2009-2010 were included. Students were followed for 14 academic terms. The results showed that the cumulative probability of dropping out in the second academic term was 0.147 . The probability of graduating within the time stipulated by the academic program was 0.187 , and 0.328 a year later. The results showed that $49.0 \%$ of the students had obtained their degree by the $14^{\text {th }}$ term and that $35.4 \%$ had dropped out. Socioeconomic variables were associated with dropping out probability while demographic conditions were associated with graduation probability. However, academic variables had a significant effect on both outcomes. It is concluded that factors associated with dropout and graduation correspond to aspects that may be intervened by educational institutions to increase student retention and graduation.
\end{abstract}




\section{INTRODUCCION}

El capital intelectual se ha convertido en uno de los activos más valiosos de la actualidad, por consiguiente, la educación, y en especial la educación terciaria, juega un papel fundamental en la medida que facilita el desarrollo pleno de las capacidades de las personas, lo que favorece el mejoramiento de su calidad de vida y sus condiciones sociales, económicas y de salud. La importancia de la educación superior impone a los países grandes retos en la medida que exige el establecimiento de sistemas educativos eficientes en términos de cobertura y calidad. La deserción en la educación se configura entonces en una de las principales fuentes de ineficiencia, ya que obstaculiza la ampliación de cobertura, genera pérdidas económicas y restringe la posibilidad de formación de recurso humano para el mercado laboral. Además de lo anterior, genera en los estudiantes y sus familias situaciones de frustración, limitación y perpetuación de ciclos de pobreza (Aljohani, 2016a, 2016b)

A nivel mundial, desde los años 70 se han desarrollado diferentes modelos teóricos para abordar el fenómeno de la deserción en educación. Concretamente, estos han sido concebidos desde cuatro perspectivas: psicológica (Fishbein y Ajzen, 1975), sociológica (Spady, 1971), económica, organizacional e interaccionista (Tinto, 1975, 1993), Independiente del enfoque, estos han coincidido en que la deserción puede ser explicada desde cuatro categorías de variables: individuales, socioeconómicas, institucionales y/o académicas (Aljohani, 2016a). No obstante, pese a la extensa investigación que se ha desarrollado alrededor del tema, hay mucho que no se sabe al respecto y que es necesario explorar para brindar evidencia empírica a los tomadores de decisiones para que puedan implementar las acciones que les permitan aumentar las tasas de finalización en los programas de formación (Aljohani, 2016b; Tinto 2006, 2010). El fenómeno de la deserción resulta complejo de abordar, ya que existen diferentes miradas del problema (individual, institucional, estatal), tipos de abandono (temprano o tardío), modos (voluntario o forzoso), lo que conlleva a que ninguna definición logre captar la complejidad del fenómeno en su totalidad. En la literatura internacional, gran parte de los estudios analizan la deserción en el primer año, debido a que es la etapa en la que se concentran las tasas más altas (Aljohani, 2016b). Sin embargo, este tipo de abordaje es limitado debido a que no permite estimar el riesgo de deserción en el tiempo, así como el comportamiento de la graduación, es decir, todo lo que sucede con el estudiante durante su paso por la universidad.

A nivel mundial, la deserción y graduación en la educación superior sigue siendo un problema de interés en todas las naciones, independente de su nivel de desarrollo. De acuerdo con las cifras reportadas por la Organización para la Cooperación y el Desarrollo Económicos (OECD, 2019), para el año 2017, en los países miembro, las tasas promedio de finalización de la educación terciaria en la duración teórica del grado fue del $39 \%$, siendo la más alta para países como Reino Unido (72\%) y la más baja para países como Chile (16\%). Las estadísticas evidencian que aún en los países más desarrollados, sólo se logran titular 7 de cada 10 estudiantes que ingresan al sistema educativo, mientras que, en países con menor grado de desarrollo esta cifra desciende a aproximadamente a 2 de cada 10. Si las cifras anteriormente descritas, se observan para la duración teórica de programa más 3 años adicionales, éstas ascienden a 67\%, 85\% y 34\% respectivamente, indicando que una buena parte de los estudiantes que se gradúan, tardan mucho más del tiempo estipulado por el programa académico, situación que trae consecuencias desfavorables para los estudiantes, sus familias, así como para las instituciones, los sistemas educativo y económico de los países. Por su parte, con respecto a la deserción del sistema educativo de los países miembro, para el 2017, la tasa promedio fue del $12 \%$ al comienzo del segundo año, del $20 \%$ para la duración teórica del programa y del $24 \%$ cuando ésta se observó tres años después del tiempo estipulado para su finalización. Las cifras más bajas se registran para países como Estados Unidos (6\%, 19\% y 20\% respectivamente), mientras que en la comunidad francesa de Bélgica, las tasas ascendieron a 21\%, 32\% y 43\%, respectivamente (OECD, 2019).

En el contexto colombiano, de acuerdo con los reportes del Ministerio de Educación Nacional de Colombia, para el año 2015 (Ministerio de Educación Nacional, 2016), la tasa de graduación a $14^{\circ}$ semestre fue del $34.5 \%$. Para el sector oficial fue del $31.1 \%$ y para el privado del $36.8 \%$. Con respecto a las áreas de conocimiento, las cifras más altas se presentaron en programas académicos pertenecientes a las ciencias de la salud (44.3\%), seguido por las ciencias de la educación (37.9\%). Para las áreas de ciencias sociales y humanas y agronomía veterinaria y afines, las estadísticas descendieron a $33.7 \%$ y $24.2 \%$ respectivamente. Con respecto a la distribución geográfica, las tasas más bajas de graduación se observaron en departamentos como Putumayo (14.9\%) y Casanare (21.6\%), en tanto que, las más altas se concentraron en Nariño (44.6\%) y Caldas (42.7\%). Para el departamento de Antioquia, donde se encuentra ubicada la ciudad de Medellín, la cifra fue del $35.1 \%$. Por su parte, el comportamiento de la deserción muestra que, para el año 2015 (Ministerio de Educación Nacional, 2016), ésta se ubicó en $46.0 \%$. Por área de conocimiento, las ciencias de la salud reportaron la cifra más baja (39.7\%), seguido por las ciencias sociales y humanas (43.6\%). Las carreras pertenecientes a agronomía veterinaria y afines exhibieron una tasa del $46.8 \%$ mientras que, la cifra para matemáticas y ciencias naturales ascendió a $52.5 \%$. Los departamentos con mayor deserción fueron Putumayo $(81.3 \%)$ y Casanare $(58.0 \%)$, mientras que, los menos afectados por la problemática fueron Huila 
(37.7\%) y Caldas (38.6\%). Antioquia se ubica un punto porcentual por encima del promedio nacional con un 47.1\%. Las cifras anteriormente descritas, evidencian que las zonas geográficas del país donde los índices de deserción y graduación son los más preocupantes, se corresponden con aquellas que tienen un menor nivel de desarrollo económico y social, así como mayores índices de desigualdad e inequidad.

En Colombia, sólo a inicios del siglo XXI, comenzó a surgir el interés por estudiar formalmente el tema de la deserción estudiantil en la educación superior. En el año 2007 (CEDE, 2007) se publicó el primer estudio de carácter nacional liderado desde el Ministerio de Educación Nacional en el que se obtuvieron datos de los estudiantes a partir de diversas fuentes de información con el fin de recopilar variables disponibles de carácter individual, socioeconómica, académica e institucional, de acuerdo con los enfoques teóricos reportados en la literatura sobre los factores explicativos de la deserción. Entre los resultados más relevantes, el estudio reportó que las características más frecuentes entre los estudiantes colombianos que desertaron se encontraban: ser hombre, tener hermanos, tener una madre con bajo nivel educativo, trabajar al momento de presentar los exámenes de estado, provenir de un hogar con bajo nivel de ingresos, haber obtenido un bajo puntaje en los exámenes de estado, tener una elevada tasa de repitencia y no recibir apoyos económicos ni académicos. Los hallazgos anteriormente descritos, dieron origen al sistema SPADIES (Sistema para la prevención y análisis de la deserción en las instituciones de educación superior), el cual permite registrar las variables anteriormente descritas y hacer seguimiento a los indicadores de deserción y graduación en estudiantes universitarios, tanto a nivel estatal como institucional.

El estado del arte sobre investigación en deserción en Colombia muestra que, durante el periodo 2006-2016 (Urrego, 2019), el 61\% de los estudios realizados han sido desarrollados bajo un enfoque cuantitativo con un abordaje predominantemente a nivel de instituciones y programas académicos. Con relación al diseño, si bien muchos de ellos trascendieron el ámbito descriptivo y buscaron explorar factores de riesgo, la mayoría lo hicieron bajo un abordaje estático el cual no permite observar el comportamiento del fenómeno desde una perspectiva longitudinal donde puedan estimarse las probabilidades deserción o graduación en momentos específicos de la trayectoria académica del estudiante. Específicamente en el contexto de la educación, resulta de gran utilidad la aplicación análisis de datos longitudinales, ya que permite analizar el tiempo que transcurre desde que el estudiante ingresa a una cohorte o un programa universitario hasta que deserta o se gradúa, así mismo, permite considerar datos censurados, que corresponde a aquellos estudiantes que al final de un determinado periodo de tiempo continúan en la universidad sin graduarse ni desertar. De acuerdo con los resultados presentados por (Urrego, 2019), en el contexto colombiano, entre 2006-2016, sólo se identificaron dos estudios con abordaje longitudinal usando modelos de supervivencia (Castaño et al., 2006; Osorio et al., 2012). Si bien este tipo de modelos han sido ampliamente utilizados desde los años 70 en diversos contextos incluyendo el educativo, es escasa su aplicación práctica por la disponibilidad de información y por la rigurosidad que implica el proceso de recolección de información por largos periodos de tiempo, bien sea de manera retrospectiva o prospectiva, como es el caso del presente estudio donde se siguieron las cohortes de estudiantes de manera retrospectiva por un periodo de 7 años.

Desde la publicación del trabajo de Cox en 1972, diversos métodos para el análisis de supervivencia se han desarrollado. Con respecto al tiempo de seguimiento, algunos se han centrado en mediciones de tipo continuo, mientras que otros lo han hecho en tiempo discreto; así mismo, frente al desenlace, algunos han considerado la ocurrencia sólo de un evento, mientras que otros, se han enfocado en modelar la existencia de múltiples eventos. En este último caso, un individuo tiene la probabilidad de experimentar más de un evento, donde la ocurrencia de uno de ellos impide la aparición de los demás, lo que se denomina riesgo competitivo. En el ámbito educativo, resulta de interés el uso de modelos de supervivencia en tiempo discreto debido a que no es posible identificar con precisión el momento exacto en el que los eventos ocurren, ya que comúnmente éstos se miden en semestres o años académicos. Así mismo, en el contexto de la educación superior, un individuo que ingresa a un programa académico puede estar en riesgo de experimentar uno de dos eventos que por naturaleza son dependientes: obtener su grado o desertar (Ortiz y Dehon, 2013). Por tanto, el hecho de que se produzca la deserción dificulta la ocurrencia de la graduación y en ese mismo sentido, un estudiante que obtiene su título académico jamás podrá experimentar la deserción. Esta situación, resulta de gran importancia, dado que, en un modelo que contemple la ocurrencia de los dos posibles eventos, la censura, sólo incluirá a aquellos estudiantes que al finalizar el periodo de seguimiento no hayan experimentado ninguno de los dos desenlaces, es decir, aquellos que persistan en la universidad.

Analizar el fenómeno bajo un modelo de supervivencia que considere la ocurrencia de sólo un evento (por ejemplo: la deserción), conllevaría a clasificar a los estudiantes que obtuvieron su grado como observaciones censuradas (Castaño et al., 2006; Osorio et al., 2012), lo que sería metodológicamente inapropiado, ya que, por definición, la censura corresponde a aquellas observaciones en las que no ocurre el evento durante el tiempo de seguimiento pero que puede ocurrir posteriormente. En este caso, un estudiante graduado, nunca tendría la probabilidad de desertar. Este abordaje, conlleva a dos dificultades. En primer lugar, a sobreestimar la probabilidad de ocurrencia del evento de interés y en segundo lugar, a no estimar adecuadamente los 
efectos de las covariables en ambos eventos, llevando a conclusiones espurias (DesJardins et al., 2002). De acuerdo con lo anterior, el análisis de supervivencia en tiempo discreto para riesgos competitivos mediante el modelo de (Scott y Kennedy, 2005) como generalización del modelo de (Singer y Willett, 1993) para K eventos de interés en un modelo logístico multinomial, se constituye en una técnica de análisis estadístico adecuada para el problema en cuestión.

El modelo de (Scott y Kennedy, 2005), ha sido utilizado en varios estudios en los últimos diez años. En una universidad de la comunidad francesa de Bélgica, realizaron un estudio con el fin de determinar factores asociados con la deserción y graduación en estudiantes de programas de pregrado de 4 años (Ortiz y Dehon, 2013). Otros investigadores, analizaron los factores que influyen en diferentes desenlaces (deserción, graduación, cambios de carrera) en una universidad italiana (Clerici et al., 2015). Otros autores, utilizaron este modelo para comparar los patrones de deserción y graduación universitaria entre minorías y mayoría de estudiantes en Noruega y Estados Unidos (Reisel y Brekke, 2010). Por su parte, en Estados Unidos desarrollaron un modelo de riesgos competitivos para determinar el efecto de la educación en línea en la deserción y la graduación (Shea y Bidjerano, 2016). Así mismo, en una universidad de Portugal analizaron la graduación y deserción entre estudiantes tradicionales y no tradicionales (Carreira y Lopes, 2019). Este modelo, también ha sido adoptado en el análisis de la deserción y graduación en programas de postgrado. En Sudáfrica, se llevó a cabo un estudio para estimar el tiempo que les toma a estos estudiantes completar con éxito o abandonar el programa de maestría y los factores que se asocian con estos desenlaces (Zewotir et al., 2015). Así mismo, en una universidad de la comunidad francesa de Bélgica, estudiaron el efecto del tipo de apoyo financiero y el campo de la investigación, en la deserción y graduación de estudiantes de doctorado (Van der Haert et al., 2014).

Para el contexto latinoamericano, no se encontró evidencia de estudios realizados mediante la aplicación del modelo de (Scott y Kennedy, 2005) donde se analice conjuntamente la deserción y la graduación, no obstante, se encontró evidencia que aborda el fenómeno de la deserción desde otras técnicas interesantes como árboles de decisión (Ramírez y Grandón, 2018), minería de datos (Miranda y Guzmán, 2017), análisis discriminante (Guerrero, 2018) y modelos logísticos (Carvajal et al., 2018). Finalmente, en el contexto colombiano, se reportaron dos estudios (Castaño et al., 2006; Osorio et al., 2012), cuyo objetivo fue analizar factores asociados a la deserción y graduación, utilizando dos modelos: el de riesgo proporcional de (Prentice y Gloeckler, 1978) que es una extensión del modelo de Cox para tiempos de duración discretos y el de (Meyer, 1990), el cual permite incluir la heterogeneidad no observable, de modo que los atributos no observables de un individuo se pueden incorporar de manera multiplicativa en la función de riesgo a partir de una variable aleatoria. Los dos modelos anteriormente mencionados, no presentaron diferencias estadísticamente significativas indicando que cuando se incluyen variables individuales, académicas, socioeconómicas e institucionales; los modelos que no incluyen heterogeneidad no observable pueden captar adecuadamente las diferencias entre los estudiantes (Castaño et al., 2006), no obstante, estos modelos tienen como limitación que no permiten combinar los dos desenlaces en un mismo modelo, lo que lleva a dificultades en el manejo de las censuras (Castaño et al., 2006; Osorio et al., 2012).

Con base en lo anterior, el presente estudio tuvo dos intencionalidades. En primer lugar, probar la hipótesis de que ciertas condiciones demográficas, socioeconómicas y académicas desfavorables, pueden estar relacionadas con una mayor probabilidad de deserción y una menor probabilidad de graduación. En segundo lugar, ilustrar la aplicación del análisis de supervivencia en tiempo discreto para riesgos competitivos usando el método de (Scott y Kennedy, 2005) que si bien no es una técnica novedosa, ha sido poco utilizada en nuestro contexto.

\section{MATERIALES Y MÉTODOS}

El diseño del estudio se realizó un estudio de seguimiento a una cohorte histórica de estudiantes nuevos en programas académicos de diez periodos de duración teórica, pertenecientes a tres áreas de conocimiento: agronomía veterinaria y afines, ciencias de la salud y ciencias sociales y humanas; registrados con matrícula entre los años 2009 y 2010, en una universidad privada de la ciudad de Medellín, a quienes se realizó seguimiento durante 14 periodos académicos. Respecto a las fuentes de información los datos fueron obtenidos a partir de los registros del sistema de admisiones de la institución y del Sistema para la prevención y análisis de la deserción en instituciones de educación superior (SPADIES). En la población de estudio se incluyeron registros de 679 estudiantes matriculados en el periodo de estudio, de los cuales, algunos fueron eliminados por completitud en los datos, quedando finalmente 639 registros para este análisis, que corresponden al $94.0 \%$ del total de estudiantes matriculados en dicho periodo.

A cada uno de los estudiantes se le realizó seguimiento por 14 periodos consecutivos. Al final del seguimiento, se obtuvo una variable dependiente con dos resultados posibles: graduado o desertor. Se identificaron como graduados a quienes obtuvieron su título académico. Como desertores, aquellos estudiantes que al final del 
seguimiento presentaron una desvinculación con una duración igual o superior a dos periodos académicos consecutivos (criterio definido por el Ministerio de Educación Nacional de Colombia y adoptado por la institución donde se realiza el estudio). Aquellos estudiantes, que al finalizar el seguimiento no presentaron ninguno de los desenlaces anteriormente descritos, se clasificaron como rezagados (censuras). En esta categoría se clasificaron los estudiantes que al final del seguimiento cumplieran con una de las siguientes tres condiciones: 1) que tuvieran matrícula activa, 2) que tuvieran una desvinculación de un semestre (inactivos), 3) que presentaran una desvinculación con una duración entre dos y cuatro periodos consecutivos pero que ya tuviera aprobadas las materias requeridas por el plan de estudios. Esta última definición se adoptó teniendo en cuenta que, estos estudiantes pueden tener una mayor probabilidad de graduarse que de desertar.

Como variables independientes, se incluyeron características demográficas, socioeconómicas y académicas consignadas en el sistema SPADIES. Dentro de las variables demográficas se incluyó el sexo y la edad. Las variables socioeconómicas fueron: estrato socioeconómico de la vivienda (clasificación en estratos de los inmuebles residenciales que deben recibir servicios públicos. Quienes tienen más capacidad económica pagan más por los servicios públicos. Por lo anterior, un mayor estrato socioeconómico, indica mayor capacidad económica), nivel educativo de la madre (si realizó o no estudios de educación superior), ingresos familiares (medidos en unidades de Salario Mínimo Mensual Legal Vigente -SMMLV-). En el grupo de variables académicas se incluyó el semestre de ingreso (si fue en el primer o segundo periodo), el puntaje estandarizado del examen de estado para ingreso a la educación superior (que evalúa las competencias de los estudiantes al finalizar la educación media, cuyos valores van en una escala de 0 a 100 donde <60 corresponde a un nivel bajo, 60 a 90 corresponde a un nivel medio y por encima de 90 se considera alto), el área de conocimiento (agrupación que se hace de los programas académicos, teniendo en cuenta afinidad en los contenidos, campos específicos del conocimiento y de acción) y la reprobación de asignaturas (si el estudiante aprobó todas las asignaturas o si reprobó al menos una).

En el estudio, fueron excluidas las variables: trabajar al momento de presentar el examen, tener hermanos, no recibir apoyos económicos ni académicos. La primera de ellas porque el $99.7 \%$ de los estudiantes no trabajaban al momento de presentar el examen y las tres restantes porque tenían subregistro superior al $20 \%$. En la figura 1, se presentan las variables tanto invariantes (aquellas características que tomaron los mismos valores a lo largo del seguimiento) como variantes en el tiempo (aquellas condiciones para las cuales se obtuvieron diferentes valores periodo a periodo).

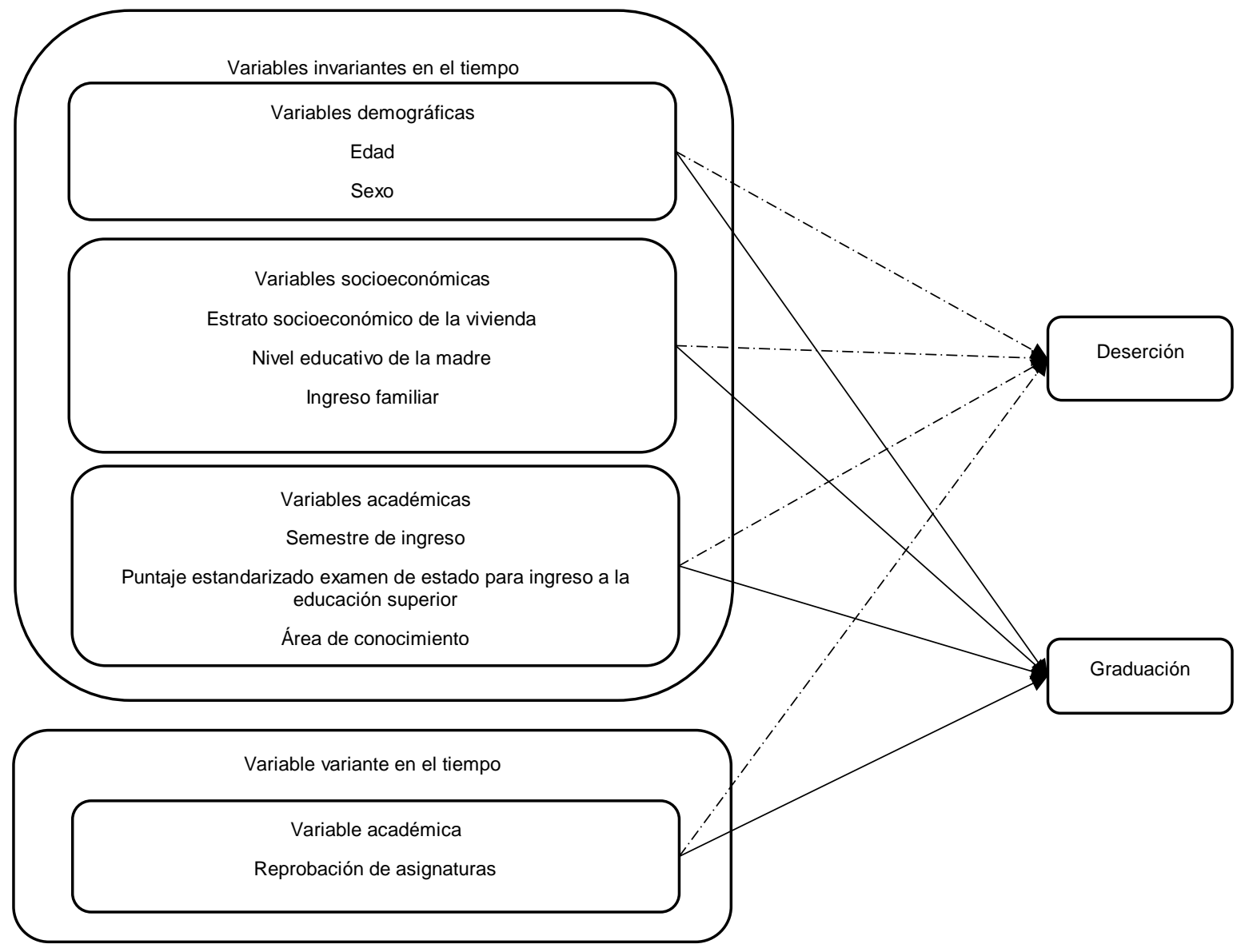

Fig. 1: Esquema de análisis de variables asociadas con la deserción y graduación 
El modelo de (Scott y Kennedy, 2005) tiene la ventaja de evaluar simultáneamente efecto de covariables tanto variantes como invariantes en la deserción y en la graduación. Para el análisis estadístico, fue necesario construir dos estructuras de datos: registros a nivel de persona y registros a nivel de persona-periodo (Singer y Willett,1993). En la primera estructura de datos, cada estudiante tenía sólo un registro en la base de datos, mientras que, en la segunda, cada estudiante tenía múltiples registros (uno por cada periodo de seguimiento con una variable indicadora de tiempo que permitió identificar el periodo dentro de cada sujeto así como el registro de los valores de la variable variante en el tiempo).

En el marco de los análisis de supervivencia, es importante determinar cómo contar el tiempo en el que el sujeto se encuentra en riesgo. En el ámbito de la educación, podría optarse por medirlo en términos de semestres inscritos o semestres transcurridos desde la matrícula inicial. La primera alternativa, puede ser de utilidad si se quiere analizar el número de semestres matriculados que un estudiante requiere para obtener su título (para determinar, por ejemplo, políticas de apoyo financiero), en tanto que, la segunda alternativa resulta de interés cuando se requiere identificar el tiempo real que toma un estudiante en obtener su título (para determinar, por ejemplo, flujos de profesionales en el mercado laboral) (Scott y Kennedy, 2005).

Para la presente investigación, el tiempo en riesgo de deserción o graduación fue medido en términos de semestres transcurridos desde la matrícula inicial. Por tanto, todos los estudiantes fueron seguidos hasta 14 periodos académicos, lo que indica que, aquellos estudiantes que en alguno de los periodos no estuvieron matriculados, igual fueron tenidos en cuenta como estudiantes en riesgo. Para este estudio se disponía de una base de datos de nivel de persona con 639 registros, la cual, al ser convertida en el formato personaperiodo quedó conformada por 5708 registros. Adicionalmente, para el modelo de riesgo en tiempo discreto, se agregaron variables dummy para cada periodo de tiempo en cada sujeto (D1 a D14). La conversión de los datos del formato persona a persona-periodo, fue realizado mediante el comando reshape del software Stata versión 16, licencia de la Universidad CES.

\section{Análisis estadístico}

La descripción de las variables independientes y el desenlace: para este componente se calcularon frecuencias y porcentajes. La función de riesgo del desenlace $k$ en el tiempo t denotada como $h(k, t)$ y función de riesgo acumulado del desenlace $K$ al tiempo t simbolizada como $M(k, t)$ se evaluaron en los desenlaces k=1: graduación; k=2: deserción, con el fin de identificar los periodos específicos en los que es más probable que los estudiantes deserten o se gradúen (Scott y Kennedy, 2005).

En un modelo de riesgos competitivos, se siguen los sujetos a lo largo de los diferentes periodos hasta que experimentan uno de los dos resultados de interés. Lo anterior supone, que sólo puede ocurrir un resultado y que, una vez que éste se produzca, el sujeto ya no está en riesgo. En el presente estudio se tienen $\mathrm{K}=2$ eventos de interés (graduación y deserción), y la no ocurrencia de ninguno de los dos eventos 0: la permanencia del estudiante en la universidad, el cual es considerado como censura. De acuerdo a (Scott y Kennedy, 2005) de la ecuación (1) se tiene que $h(k, t)$ es una probabilidad condicional y se define como la probabilidad de que el evento $k$ ocurra en el tiempo $t$, dado que ninguno de los dos eventos $(k=0)$ ha sucedido en cada período anterior a t. Lo anterior indica que la probabilidad de ambos resultados (deserción 0 graduación), se estimará con relación a la probabilidad de que el estudiante no experimente ningún desenlace (es decir, que permanezca matriculado en la universidad).

$$
\mathrm{h}(\mathrm{k}, \mathrm{t})=\frac{\# \text { sujetos que experimentan el desenlace } \mathrm{k} \text { en el periodo } \mathrm{t}}{\# \text { sujetos a riesgo en el periodo } \mathrm{t}}
$$

Note en la ecuación (2) que $h(t)$ es el riesgo de que cualquier desenlace ocurra en el tiempo t:

$$
h(t)=\sum_{k} h(k, t)
$$

De la ecuación (3) se tiene que $M(k, t)$ se define como la probabilidad acumulada de que $k$ ocurra en los primeros t periodos, por lo tanto $\mathrm{M}(\mathrm{t})$ es la suma de las probabilidades acumuladas variando $\mathrm{k}=1,2$ para este caso graduación o deserción:

$$
M(t)=\sum_{k} M(k, t)
$$

Luego, $1-M(t)$ es la función de supervivencia y $h(k, t)[1-M(t-1)]$ es la probabilidad de que $K$ ocurra en el periodo t. Las expresiones para $M(t)$ y $M(k, t)$ se presentan las ecuaciones (4) y (5). Para $M(k, t)$ se tiene que: 


$$
\begin{aligned}
& M(k, 1)=h(k, 1) \\
& M(k, t)=h(k, t)[1-M(t-1)]+M(k, t-1) \text { para } t>1
\end{aligned}
$$

Para $M(t)$ se tiene que:

$$
\begin{aligned}
& M(1)=\sum_{k} h(k, 1) ; \\
& M(t)=\left[\sum_{k} h(k, t)\right][1-M(t-1)]+M(t-1) \text { para } t>1
\end{aligned}
$$

En el Modelo en tiempo discreto para riesgos competitivos las funciones de riesgo y de riesgo acumulado descritas en el numeral 2), permiten identificar en qué momento de la trayectoria académica es más probable que los estudiantes deserten o finalicen con éxito su formación académica, sin embargo, no permiten medir el efecto que ciertas variables de interés puedan tener en la probabilidad de deserción y graduación.

En respuesta a lo anterior, el modelo en tiempo discreto para riesgos competitivos propuesto por (Scott y Kennedy, 2005) permite ajustar dichas probabilidades por ciertas características de interés. Este modelo, se basa en la regresión logística multinomial para estimar el riesgo. Los modelos logísticos multinomiales tienen una interpretación similar a los modelos logísticos simples. En este caso, un cambio unitario en la variable $X_{j}$, tiene el efecto de multiplicar por $\exp \left(\beta_{\mathrm{kj}}\right)$ a la razón de riesgos relativo $(\mathrm{RRR}) \mathrm{h}(\mathrm{k}, \mathrm{t}) / \mathrm{h}(0, \mathrm{t})$. Si $\beta_{\mathrm{kj}}>0$ entonces un incremento en $X_{j}$, incrementará la razón de riesgos del evento k (Scott y Kennedy, 2005; Singer y Willett, 1993).

El Modelo de línea base se obtiene cuando no se introducen covariables en el modelo (ecuación (6)). Este permite identificar el riesgo de ocurrencia del evento en cada periodo de tiempo (Ortiz y Dehon, 2013; Singer y Willett, 1993):

$$
\log \left[\frac{h_{i}(k, t)}{h_{i}(0, t)}\right]=\left[\alpha_{k 1} D_{i 1}+a_{k 2} D_{i 2}+\ldots+\alpha_{k T} D_{i T}\right], k \in\{1, \ldots, K\}, i \in\{1, \ldots, n\}, t \in\{1, \ldots, T\}
$$

Donde $h_{i}(k, t)$ es el riesgo de que suceda el resultado $k$ en el tiempo t para el sujeto $i . h_{i}(0, t)$ es el riesgo de que ningún evento ocurra. $h_{i}(k, t) / h_{i}(0, t)$ se conoce como la razón de riesgos de desenlace específico debido a que mide el riesgo relativo de experimentar el evento $\mathrm{k}$ con respecto al riesgo de no presentar ningún evento. $\left[\mathrm{D}_{1 \mathrm{it}}, \ldots, \mathrm{D}_{\mathrm{Tit}}\right]$ son variables dummy que actúan como múltiples interceptos, una por periodo de tiempo, donde $\mathrm{T}$ representa el último periodo observado para un sujeto. Por ejemplo, si un estudiante fue observado durante 3 periodos tendrá tres variables dummy así: en el primer periodo $\left[D_{1 i 1}=1, D_{2 i 1}=0, D_{3 i 1}=0\right.$ ], en el segundo periodo $\left[D_{1 i 2}=0, D_{2 i 2}=1, D_{3 i 2}=0\right]$ y en el tercer periodo $\left[D_{1 i 3}=0, D_{2 i 3}=0, D_{3 i 3}=1\right] .\left[\alpha_{k 1, \ldots,} a_{k T}\right]$ capturan el nivel de riesgo de línea de base en cada período de tiempo. La estimación de este modelo de referencia nos permite ver cómo el modelo logístico multinomial se usa como una herramienta estadística para establecer una relación entre el riesgo y las covariables (Ortiz y Dehon, 2013).

El Modelo con covariables es un modelo estadístico, tiene dos componentes: (a) un perfil de riesgo de línea base y (b) un conjunto de parámetros que capturan el efecto de las variables independientes sobre la razón de riesgos del evento $\mathrm{k}$ en relación con no haber presentado ningún evento. b) un conjunto de parámetros que capturan el efecto de las variables independientes. De acuerdo con lo anterior, el modelo propuesto en la ecuación (7) para el sujeto $i \in\{1, \ldots, n\}$ del desenlace $k \in\{1, \ldots, K\}$ en el tiempo $t \in\{1, \ldots, T\}$ es (Ortiz y Dehon, 2013; Singer y Willett, 1993):

$$
\log \left[\frac{h_{i}(k, t)}{h_{i}(0, t)}\right]=\left[\alpha_{k 1} D_{i 1}+\alpha_{k 2} D_{i 2}+\ldots+\alpha_{k T} D_{i T}\right]+\left[\beta_{k 1} X_{1 i t}+\ldots+\beta_{k p} X_{p i t}\right]
$$

Donde, $h_{i}(k, t)$ es el riesgo de que suceda el resultado $k$ en el tiempo t para el sujeto i. Esta función de riesgo incluye covariables, los parámetros de regresión asociados a cada covariable y el resultado. $\left[\mathrm{X}_{1}, \ldots, \mathrm{X}_{\mathrm{p}}\right]$ son las $p$ covariables incluidas en el modelo, las cuales pueden ser variantes o invariantes en el tiempo, lo que explica el hecho $X$ esté indexado en el tiempo $t$ (Singer y Willett, 1993). [ $\left.\beta_{\mathrm{k} 1}, \ldots, \beta_{\mathrm{kT}}\right]$ describen los efectos de las $p$ variables independientes sobre la razón de riesgo del evento k (en una escala logit). En lo que sigue se describen los supuestos de linealidad, no heterogeneidad no inobservada y proporcionalidad.

La Linealidad es un supuesto que establece que, diferencias iguales en los valores de las variables independientes corresponden a desplazamientos verticales iguales del perfil logit-hazard. Para la presente investigación, este supuesto no es relevante debido a que el conjunto completo de variables independientes está compuesto por variables dummy (Ortiz y Dehon, 2013; Singer y Willett, 1993). 
La no heterogeneidad inobservada es un supuesto establece que, el modelo no requiere un término de error debido a que toda la heterogeneidad entre los individuos se explica por la variación en los valores de las covariables. El problema con este supuesto radica en que la omisión de variables importantes en el modelo puede llevar a problemas en las estimaciones. Sin embargo, los posibles sesgos disminuyen cuando se utiliza una especificación flexible para la función de riesgo de referencia, como es el caso del presente modelo (Ortiz y Dehon, 2013; Singer y Willett, 1993).

La Proporcionalidad es un supuesto que establece que la familia de perfiles logit hazard representada por todos los valores posibles de las covariables comparten una forma común y son mutuamente paralelos, siendo separados solo verticalmente por los diferentes valores de los predictores (por ejemplo, para hombres y mujeres). Para validar este supuesto, se pueden graficar los perfiles logit hazard a lo largo del tiempo para cada una de las categorías de la variable independiente; si éstas son aproximadamente paralelas y no se cruzan, puede concluirse que el supuesto se cumple (Singer y Willett, 1993).

Para la validación del supuesto de riesgos proporcionales en este modelo, se graficaron los perfiles logit Hazard a lo largo del tiempo para cada una de las variables independientes (tanto variantes como invariantes en el tiempo) de dos formas: sin interacción y en interacción con las dummy del tiempo (fórmulas 8 y 9 ). El abordaje anteriormente descrito, se hace desde una perspectiva más escéptica, partiendo del supuesto de que no se cumpla el supuesto de proporcionalidad; por tanto, si para una variable, ambas curvas son similares para los dos desenlaces, se confirma que el supuesto de riesgos proporcionales se cumple; en caso contrario, éste es violado y por consiguiente la variable debe ser incluida en el modelo en interacción con el tiempo (Singer y Willett, 1993). En la ecuación (8) se presenta el modelo donde el efecto de una variable independiente $X_{j}$, interactúa con el tiempo:

$$
\log \left[\frac{h_{i}(k, t)}{h_{i}(0, t)}\right]=\left[\alpha_{k 1} D_{i 1}+\alpha_{k 2} D_{i 2}+\ldots+\alpha_{k T} D_{i T}\right]+\left[\beta_{1 k j}\left(D_{i 1} \times X_{j i t}\right)+\ldots+\beta_{T k j}\left(D_{i T} \times X_{j i t}\right)\right]
$$

En la ecuación (9) se presenta el modelo donde el efecto de una variable independiente $X_{j}$, no interactúa con el tiempo:

$$
\log \left[\frac{h_{i}(k, t)}{h_{i}(0, t)}\right]=\left[\alpha_{k 1} D_{i 1}+\alpha_{k 2} D_{i 2}+\ldots+\alpha_{k T} D_{i T}\right]+\left[\beta_{k j} x_{j i t}\right]
$$

En el presente estudio, primero se calculó el modelo de línea base, posteriormente, para cada una de las variables independientes se evaluó el supuesto de proporcionalidad, para determinar si debían incluirse en el modelo con o sin interacción con el tiempo. Una vez identificado el comportamiento de cada una de las variables, se estimaron las medidas tanto crudas como ajustadas por todas las variables. Para la estimación el modelo, se utilizó el comando mlogit, del paquete estadístico Stata versión 16, licencia de la Universidad CES. Para las gráficas se utilizó Microsoft Excel. En cuanto a aspectos éticos, de acuerdo con lo establecido en el Artículo 11 de la Resolución 008430 de 1993 del Ministerio de Salud de Colombia, esta investigación se clasificó como sin riesgo debido a que utilizó información obtenida a partir de fuentes secundarias.

\section{RESULTADOS}

Se analizaron los registros de 639 estudiantes nuevos matriculados en las cohortes 2009 y 2010 , que ingresaron a programas pertenecientes a las siguientes áreas de conocimiento: Agronomía, veterinaria y afines (29.7\%), Ciencias de la salud (49.3\%) y Ciencias sociales y humanas (21.0\%). El $72.3 \%$ eran mujeres, el $57.4 \%$ tenían 18 años o más, el $62.4 \%$ tenían una madre con nivel educativo superior, el $47.6 \%$ habitaba viviendas en estratos entre 4 y 6 , el 35.2\% tenía ingresos familiares superiores a los 5 salarios mínimos mensuales legales vigentes (SMMLV), el $62.3 \%$ ingresó en el primer semestre del año, el $74.8 \%$ obtuvo un puntaje en el examen de Estado para el ingreso a la educación superior medio o alto y el $48.0 \%$ reprobó al menos una asignatura en el primer periodo (Tabla 3).

El comportamiento del desenlace por sexo muestra que, de cada 100 mujeres que ingresaron se graduaron 53; en tanto que, de cada 100 hombres se graduaron 40. Con relación a la edad, se observa que de cada 100 menores de 18 años desertaron 33; mientras que, en el grupo de estudiantes de 18 años o más, desertaron 37 de cada 100. Por su parte, las características socioeconómicas muestran que, la proporción de estudiantes graduados aumentó a medida que el estrato socioeconómico de sus viviendas y el ingreso familiar también lo hicieron, en tanto que, la proporción de desertores disminuyó cuando estas condiciones eran mejores. 
De acuerdo con las características académicas, el comportamiento del desenlace por semestre muestra que de cada 100 estudiantes que ingresaron en el primer periodo del año, se graduaron 53, comparado con 42 para los que ingresaron en el segundo periodo. El puntaje de examen de Estado para el ingreso a la educación superior muestra que a medida que éste aumenta, la graduación aumenta y la deserción disminuye. Finalmente, el área de conocimiento muestra una mayor proporción de estudiantes graduados para las ciencias sociales, en tanto que, Agronomía, veterinaria y afines fue la que presentó una mayor proporción de estudiantes desertores. La reprobación de asignaturas en el primer periodo muestra que, el $48.0 \%$ de los estudiantes experimentaron la reprobación de al menos una asignatura cuando iniciaron su carrera, entre estos estudiantes, se observó una deserción del 58.0\%. Por su parte, entre los estudiantes que aprobaron todas sus asignaturas en el primer periodo, el 74.4\% obtuvieron su grado (Tabla 3).

Tabla 3. Descripción de las características demográficas, socioeconómicas y académicas de los estudiantes universitarios según desenlace

\begin{tabular}{|c|c|c|c|c|c|c|}
\hline Variable & Categoría & $n$ & $\%$ & Deserción & Graduación & Censura \\
\hline \multirow[t]{2}{*}{ Sexo } & Mujer & 462 & $72.3 \%$ & $33.5 \%$ & $52.6 \%$ & $13.9 \%$ \\
\hline & Hombre & 177 & $27.7 \%$ & $40.1 \%$ & $39.5 \%$ & $20.3 \%$ \\
\hline \multirow{2}{*}{ Edad } & $<18$ & 272 & $42.6 \%$ & $33.1 \%$ & $54.0 \%$ & $12.9 \%$ \\
\hline & $>=18$ & 367 & $57.4 \%$ & $37.1 \%$ & $45.2 \%$ & $17.7 \%$ \\
\hline \multirow[t]{2}{*}{ Nivel educativo de la madre } & Educación técnica o inferior & 240 & $37.6 \%$ & $46.3 \%$ & $37.1 \%$ & $16.7 \%$ \\
\hline & Educación superior & 399 & $62.4 \%$ & $28.8 \%$ & $56.1 \%$ & $15.0 \%$ \\
\hline \multirow{3}{*}{$\begin{array}{l}\text { Estrato socioeconómico de la } \\
\text { vivienda }\end{array}$} & $1-3$ & 335 & $52.4 \%$ & $43.3 \%$ & $42.1 \%$ & $14.6 \%$ \\
\hline & $4-5$ & 198 & $31.0 \%$ & $29.8 \%$ & $51.5 \%$ & $18.7 \%$ \\
\hline & 6 & 106 & $16.6 \%$ & $20.8 \%$ & $66.0 \%$ & $13.2 \%$ \\
\hline \multirow{3}{*}{ Ingreso familiar } & $<2$ SMMLV & 130 & $20.3 \%$ & $51.5 \%$ & $33.8 \%$ & $14.6 \%$ \\
\hline & 2 - 5 SMMLV & 284 & $44.4 \%$ & $34.2 \%$ & $48.6 \%$ & $17.3 \%$ \\
\hline & $>5$ SMMLV & 225 & $35.2 \%$ & $27.6 \%$ & $58.2 \%$ & $14.2 \%$ \\
\hline \multirow[t]{2}{*}{ Semestre de ingreso } & $\mathrm{I}$ & 398 & $62.3 \%$ & $32.9 \%$ & $53.0 \%$ & $14.1 \%$ \\
\hline & II & 241 & $37.7 \%$ & $39.4 \%$ & $42.3 \%$ & $18.3 \%$ \\
\hline \multirow{3}{*}{ Puntaje examen de Estado } & Bajo & 161 & $25.2 \%$ & $55.9 \%$ & $24.8 \%$ & $19.3 \%$ \\
\hline & Medio & 287 & $44.9 \%$ & $35.5 \%$ & $47.4 \%$ & $17.1 \%$ \\
\hline & Alto & 191 & $29.9 \%$ & $17.8 \%$ & $71.7 \%$ & $10.5 \%$ \\
\hline \multirow[t]{3}{*}{ Área de conocimiento } & $\begin{array}{l}\text { Agronomía, veterinaria y } \\
\text { afines }\end{array}$ & 190 & $29.7 \%$ & $45.3 \%$ & $28.9 \%$ & $25.8 \%$ \\
\hline & Ciencias de la salud & 315 & $49.3 \%$ & $32.7 \%$ & $54.6 \%$ & $12.7 \%$ \\
\hline & Ciencias sociales y humanas & 134 & $21.0 \%$ & $27.6 \%$ & $64.2 \%$ & $8.2 \%$ \\
\hline \multirow{2}{*}{$\begin{array}{l}\text { Reprobación de asignaturas (Periodo } \\
\text { 1) }\end{array}$} & Si & 307 & $48.0 \%$ & $58.0 \%$ & $21.5 \%$ & $20.5 \%$ \\
\hline & No & 332 & $52.0 \%$ & $14.5 \%$ & $74.4 \%$ & $11.1 \%$ \\
\hline
\end{tabular}

Con respecto al desenlace, se encontró que el $35.4 \%$ desertaron, el $49.0 \%$ se graduaron y el $15.6 \%$ no presentó ninguno de ellos. Entre los desertores, 54 de cada 100 abandonaron su carrera en los tres primeros periodos académicos y sólo 11 de ellos lo hizo después del periodo 7. Para la graduación, sólo 1 de cada 4 estudiantes graduados logró obtener su título en el tiempo establecido por el programa académico, así mismo, 25 de cada 100 se graduó en el año siguiente después de haber completado la duración teórica del programa (Tabla 4).

Tabla 4. Descripción de la graduación o deserción de los estudiantes universitarios

\begin{tabular}{|l|c|c|}
\hline Variable & $n$ & $\%$ \\
\hline Deserción & 226 & $35.4 \%$ \\
\hline Primeros 3 periodos & 122 & $54.0 \%$ \\
\hline Periodos 4 a 7 & 79 & $35.0 \%$ \\
\hline Después del periodo 7 & 25 & $11.1 \%$ \\
\hline Graduación & 313 & $49.0 \%$ \\
\hline En el tiempo & 79 & $25.2 \%$ \\
\hline 1 periodo después & 71 & $22.7 \%$ \\
\hline 2 periodos después & 85 & $27.2 \%$ \\
\hline 3 periodos después & 43 & $13.7 \%$ \\
\hline 4 periodos después & 35 & $11.2 \%$ \\
\hline Censura & 100 & $15.6 \%$ \\
\hline Total & 639 & $100.0 \%$ \\
\hline
\end{tabular}

Para la estimación de la Función de riesgo del desenlace k en el tiempo t y la Función de riesgo acumulado del desenlace al tiempo t : en la tabla 5, se presenta el comportamiento de la función de riesgo y la función de riesgo acumulada de deserción y graduación. Con respecto a la deserción, se puede apreciar que se presentó entre los periodos 1 y 12. De los 639 estudiantes que estuvieron a riesgo el primer periodo, 63 
desertaron. Por tanto, la probabilidad de desertar en el primer periodo fue de 0.099. Para el segundo periodo, la probabilidad de desertar fue de 0.054. A lo largo de los diferentes semestres, se puede observar que la función de riesgo tiende a disminuir a medida que avanzan los periodos académicos, aunque se observan dos incrementos en los periodos cuatro y nueve. La función de riesgo acumulada muestra que para el semestre 12, el $35.4 \%$ de los estudiantes de la cohorte había desertado (Tabla 5).

Para la graduación, se observa que ésta se presentó a partir del periodo 10. La función de riesgo para este periodo muestra que de los 423 estudiantes que estuvieron a riesgo, dado que no habían experimentado ninguno de los dos eventos en el periodo anterior, 79 se graduaron. Por tanto, la probabilidad de graduación en este periodo fue de 0.187 . El periodo donde resultó más alta la probabilidad de graduarse fue en el 12 con un valor de 0.321, un año después del tiempo estipulado por el programa académico. La función de riesgo acumulada muestra que para el periodo 14 , el $49 \%$ de los estudiantes de la cohorte habían obtenido su título académico (Tabla 5). La función de riesgo acumulada total indica que para el periodo 14 , el $84.4 \%$ de los estudiantes había experimentado uno de los dos desenlaces, deserción o graduación. La mediana de la supervivencia fue de aproximadamente 11 periodos, lo que indica que el $50.0 \%$ de los estudiantes experimentaron uno de los dos desenlaces antes de este periodo y el otro $50.0 \%$ lo experimentó después. La función de riesgo acumulada muestra que para el periodo 12, las probabilidades acumuladas de desertar y graduarse eran similares (Tabla 5).

Tabla 5. Función de riesgo y función de riesgo acumulada de deserción y graduación

\begin{tabular}{|l|l|l|l|l|l|l|l|l|l|l|l|l|}
\hline Periodo & Población en riesgo & \multicolumn{2}{l|}{ Frecuencia } & \multicolumn{3}{l|}{ Función de riesgo } & \multicolumn{3}{l|}{ Función de riesgo acumulada } \\
\cline { 3 - 12 } & & Des & Grad & Censura & Des & Grad & $\begin{array}{l}\text { Total } \\
(t)\end{array}$ & & & & Ges & \\
\hline 1 & 639 & 63 & 0 & 0 & 0.099 & 0.000 & 0.099 & 0.099 & 0.000 & 0.099 \\
\hline 2 & 576 & 31 & 0 & 0 & 0.054 & 0.000 & 0.054 & 0.147 & 0.000 & 0.147 \\
\hline 3 & 545 & 28 & 0 & 0 & 0.051 & 0.000 & 0.051 & 0.191 & 0.000 & 0.191 \\
\hline 4 & 517 & 32 & 0 & 0 & 0.062 & 0.000 & 0.062 & 0.241 & 0.000 & 0.241 \\
\hline 5 & 485 & 21 & 0 & 0 & 0.043 & 0.000 & 0.043 & 0.274 & 0.000 & 0.274 \\
\hline 6 & 464 & 15 & 0 & 0 & 0.032 & 0.000 & 0.032 & 0.297 & 0.000 & 0.297 \\
\hline 7 & 449 & 11 & 0 & 0 & 0.024 & 0.000 & 0.024 & 0.315 & 0.000 & 0.315 \\
\hline 8 & 438 & 5 & 0 & 0 & 0.011 & 0.000 & 0.011 & 0.322 & 0.000 & 0.322 \\
\hline 9 & 433 & 10 & 0 & 0 & 0.023 & 0.000 & 0.023 & 0.338 & 0.000 & 0.338 \\
\hline 10 & 423 & 6 & 79 & 0 & 0.014 & 0.187 & 0.201 & 0.347 & 0.124 & 0.471 \\
\hline 11 & 338 & 2 & 71 & 0 & 0.006 & 0.210 & 0.216 & 0.351 & 0.235 & 0.585 \\
\hline 12 & 265 & 2 & 85 & 0 & 0.008 & 0.321 & 0.328 & 0.354 & 0.368 & 0.721 \\
\hline 13 & 178 & 0 & 43 & 0 & 0.000 & 0.242 & 0.242 & 0.354 & 0.435 & 0.789 \\
\hline 14 & 135 & 0 & 35 & 100 & 0.000 & 0.259 & 0.259 & 0.354 & 0.490 & 0.844 \\
\hline Total & 639 & 226 & 313 & 100 & 0.354 & 0.490 & 0.844 & & & \\
\hline
\end{tabular}

Con respecto al comportamiento de la función de riesgo acumulada de deserción y graduación por área de conocimiento, se pudo determinar que, la deserción en los tres primeros periodos académicos fue similar en tres áreas estudiadas, no obstante, a partir del cuarto periodo se observan diferencias entre ellas, principalmente para agronomía, veterinaria y afines donde la cifra fue del $45.3 \%$ para el periodo 14 , comparada con un $32.7 \%$ para ciencias de la salud y $27.6 \%$ para ciencias sociales y humanas. Con respecto a la graduación, la probabilidad de obtener el título en el tiempo fue del $18.7 \%$ en los programas pertenecientes a ciencias sociales y humanas, $15.6 \%$ para ciencias de la salud y sólo del $2.6 \%$ para agronomía veterinaria y afines. Para el periodo 14 la mayor tasa de graduación se obtuvo para programas pertenecientes al área de ciencias sociales y humanas con un $64.2 \%$, mientras que, para los pertenecientes a Agronomía, veterinaria y afines ésta fue del $28.9 \%$. Se resalta que, para esta última área de conocimiento, para el periodo 14 sólo el $74,2 \%$ de los estudiantes había experimentado uno de los dos desenlaces y el $25.8 \%$ aún permanecía en la universidad (Figura 2)

El comportamiento de la función de riesgo acumulada de deserción y graduación por semestre de ingreso muestra que la deserción es mayor entre los estudiantes que iniciaron sus estudios en el segundo semestre. Para el periodo 14, la tasa de deserción fue del $32.9 \%$ entre los estudiantes que ingresaron en el primer periodo, mientras, que entre los que lo hicieron en el segundo periodo, esta cifra ascendió a 39.4\%. Para la graduación se observa que, mientras el $19.8 \%$ de los estudiantes que ingresaron en el primer semestre, obtuvieron su título en el tiempo, ninguno de los que ingresó en el segundo periodo pudo hacerlo. Si bien para el periodo 11, se observa que la probabilidad acumulada de graduación es levemente superior entre los estudiantes que ingresaron en el segundo semestre (24.9\% vs $22.6 \%$ ), para el resto de los periodos, sigue siendo inferior llegando a ser del $42.3 \%$ para el periodo 14 comparado con un $53.0 \%$ para los estudiantes que ingresaron en el primer periodo (Figura 3). 


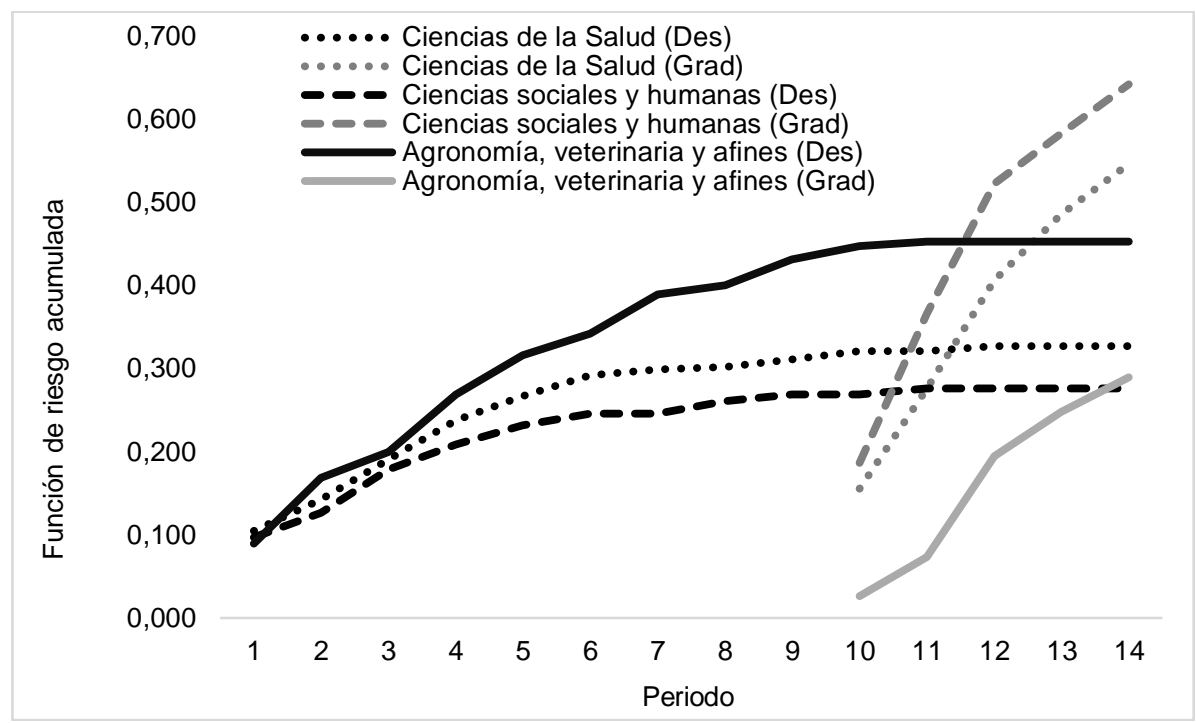

Fig. 2: Función de riesgo acumulada de deserción y graduación por área de conocimiento

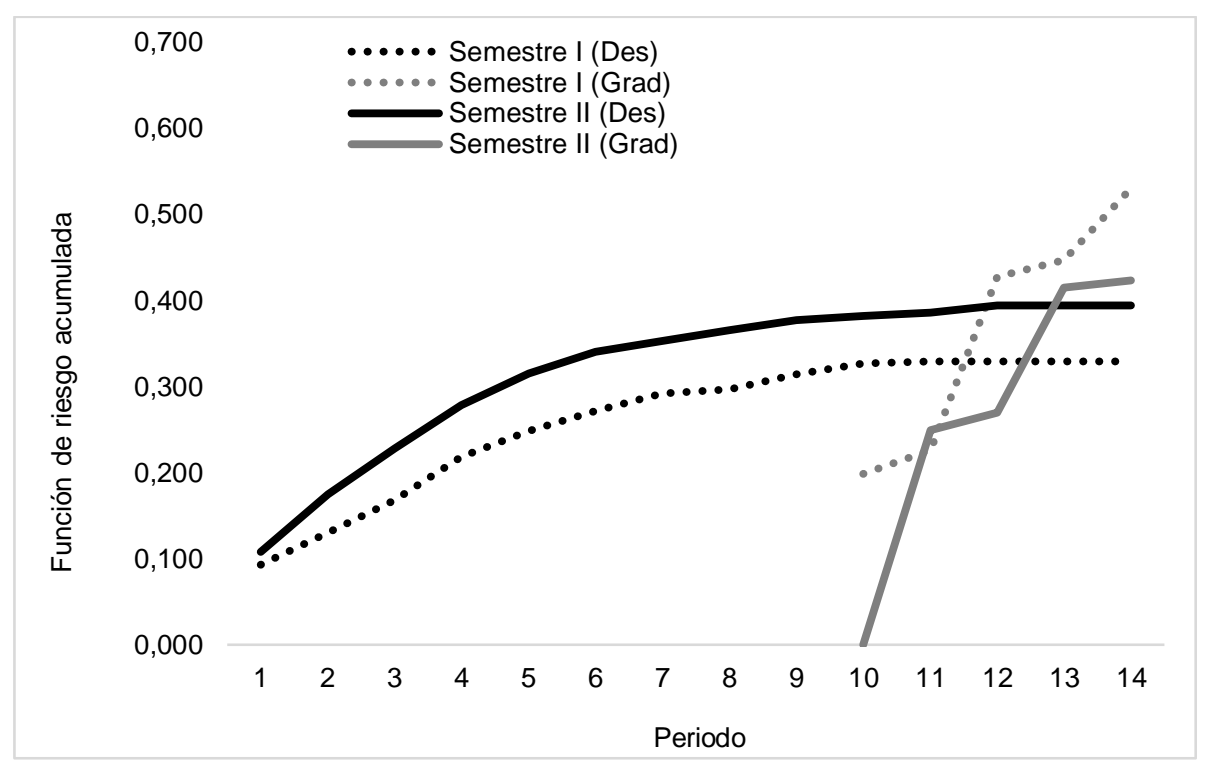

Fig. 3: Función de riesgo acumulada de deserción y graduación por semestre de ingreso

\section{Modelo en tiempo discreto para riesgos competitivos}

En la tabla 6 se presentan los resultados obtenidos con el modelo de línea base. La razón de riesgo relativo (RRR) muestra que, para el periodo 1 el riesgo relativo de desertar era 0.109 veces el riesgo relativo de no presentar ningún evento para este mismo periodo. Así mismo, para el periodo 12 el riesgo relativo de graduarse era 0.528 veces el riesgo relativo de no presentar ningún evento para este mismo periodo. Para los 12 periodos en los que ocurrieron deserciones y para los cinco periodos en los que los estudiantes obtuvieron su grado, los coeficientes fueron estadísticamente significativos (valor $p<0.05$ ).

Para la validación del supuesto de proporcionalidad: previo a la estimación del modelo, se procedió con la validación del supuesto para cada una de las variables independientes. A excepción del semestre de ingreso, todas cumplieron con el supuesto de riesgos proporcionales. En la figura 4 se observa que la función de riesgo acumulada para deserción en los hombres tiene un comportamiento similar cuando se incluye y se omite la interacción con el tiempo (linea negra punteada y continua respectivamente). Este mismo comportamiento se observa para las mujeres (lineas grises). Las curvas para hombres y mujeres (lineas punteadas negras y grises), permiten evidenciar que el supuesto se cumple ya que éstas son aproximadamente paralelas y no se cruzan. El comportamiento descrito anteriormente, también se observa para la graduación (Figura 5). 
Tabla 6. Ajuste del modelo de línea base. * Estadísticamente significativo a nivel de 0.05

\begin{tabular}{|c|c|c|c|c|c|c|}
\hline \multirow{2}{*}{ Periodo } & \multicolumn{5}{|c|}{ Deserción } & \multicolumn{3}{c|}{ Graduación } \\
\cline { 2 - 6 } & Coeficiente & RRR (IC95\%) & Valor $p$ & Coeficiente & RRR (IC95\%) & Valor $p$ \\
\hline 1 & -2.213 & $0.109(0.084 ; 0.142)$ & $<0.001^{*}$ & & & \\
\hline 2 & -2.852 & $0.058(0.040 ; 0.083)$ & $<0.001^{*}$ & & & \\
\hline 3 & -2.898 & $0.055(0.038 ; 0.081)$ & $<0.001^{*}$ & & & \\
\hline 4 & -2.678 & $0.069(0.048 ; 0.098)$ & $<0.001^{*}$ & & & \\
\hline 5 & -3.069 & $0.046(0.030 ; 0.072)$ & $<0.001^{*}$ & & & \\
\hline 6 & -3.376 & $0.034(0.020 ; 0.057)$ & $<0.001^{*}$ & & & \\
\hline 7 & -3.671 & $0.025(0.014 ; 0.046)$ & $<0.001^{*}$ & & & \\
\hline 8 & -4.436 & $0.012(0.005 ; 0.029)$ & $<0.001^{*}$ & & & \\
\hline 9 & -3.726 & $0.024(0.013 ; 0.045)$ & $<0.001^{*}$ & & & \\
\hline 10 & -4.004 & $0.018(0.008 ; 0.041)$ & $<0.001^{*}$ & -1.427 & $0.240(0.188 ; 0.307)$ & $<0.001^{*}$ \\
\hline 11 & -4.779 & $0.008(0.002 ; 0.034)$ & $<0.001^{*}$ & -1.210 & $0.298(0.229 ; 0.389)$ & $<0.001^{*}$ \\
\hline 12 & -4.388 & $0.012(0.003 ; 0.050)$ & $<0.001^{*}$ & -0.639 & $0.528(0.406 ; 0.687)$ & $<0.001^{*}$ \\
\hline 13 & & & -0.984 & $0.374(0.263 ; 0.531)$ & $<0.001^{*}$ \\
\hline 14 & & & -0.814 & $0.443(0.298 ; 0.660)$ & $<0.001^{*}$ \\
\hline
\end{tabular}

Contrario a lo anterior, si bien el semestre de ingreso del estudiante cumple con el supuesto de riesgos proporcionales para la deserción (Figura 6), no lo cumple para la graduación. Las líneas punteadas (negra y gris) de la figura 7 muestran que si las curvas se obtienen sin considerar la interacción de la variable con el tiempo, éstas son aproximadamente paralelas y no se cruza, lo que llevaría a asumir erroneamente que se cumple el supuesto, no obstante, cuando se grafican las curvas considerando la interacción de la variable con el tiempo (lineas continuas gris y negra), éstas no son paralelas y además se cruzan.

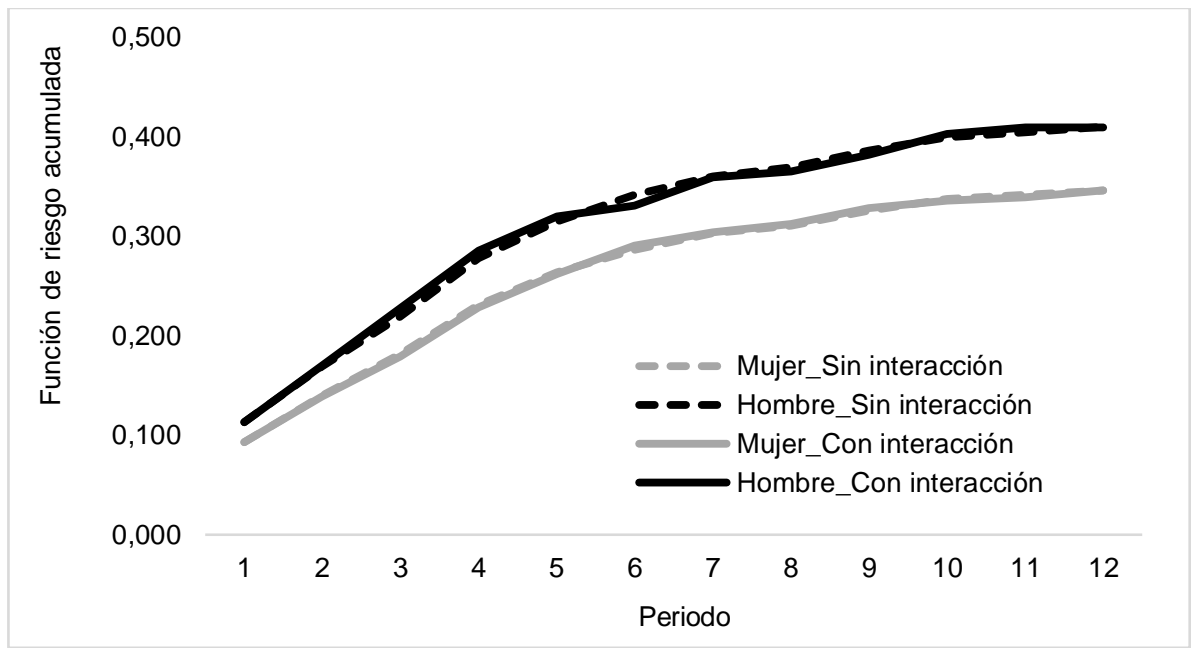

Fig. 4: Función de riesgo acumulada de deserción según sexo con y sin interacción con el tiempo

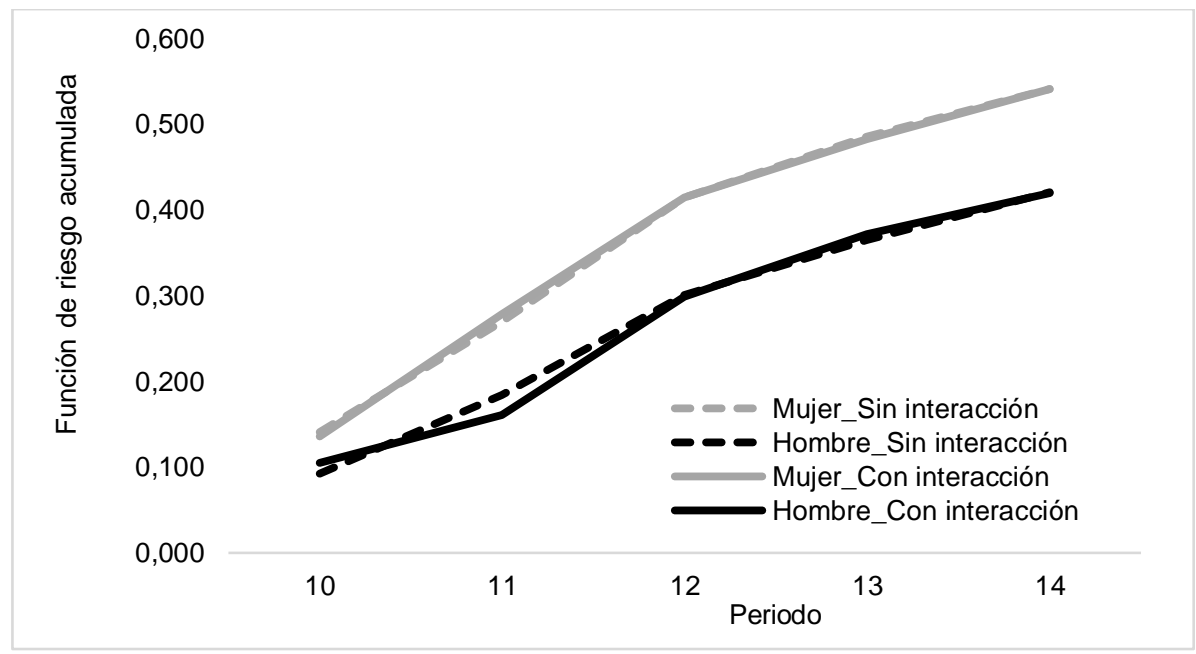

Fig. 5: Función de riesgo acumulada de graduación según sexo con y sin interacción con el tiempo 


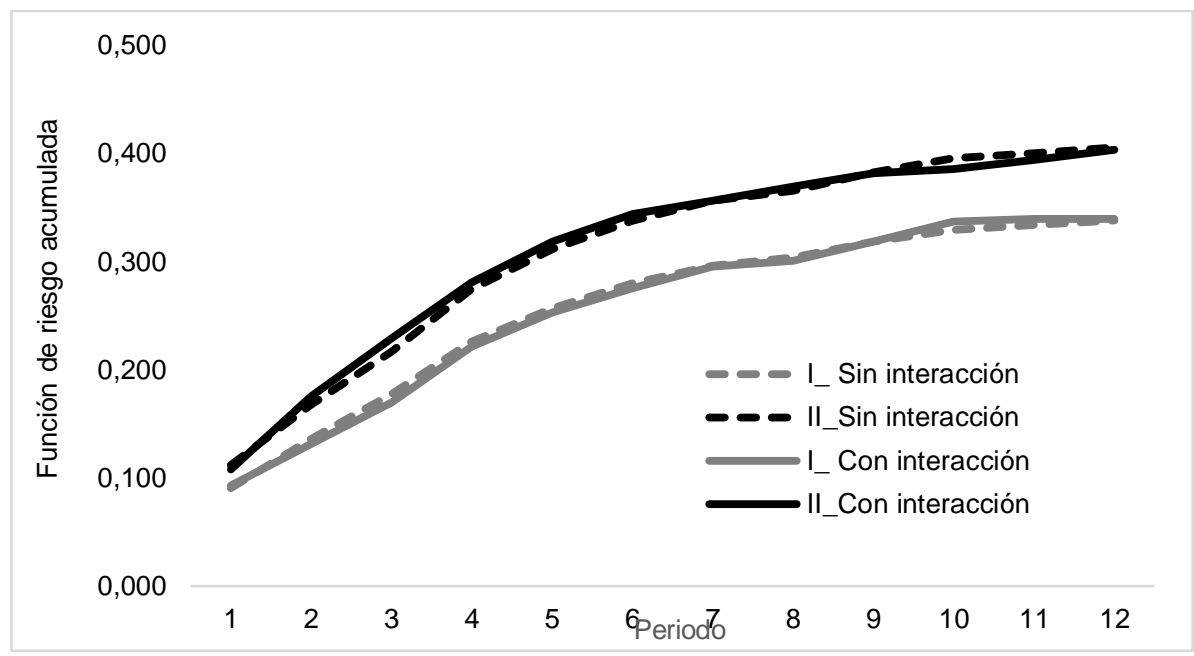

Fig. 6: Función de riesgo acumulada de deserción según semestre de ingreso con y sin interacción con el tiempo

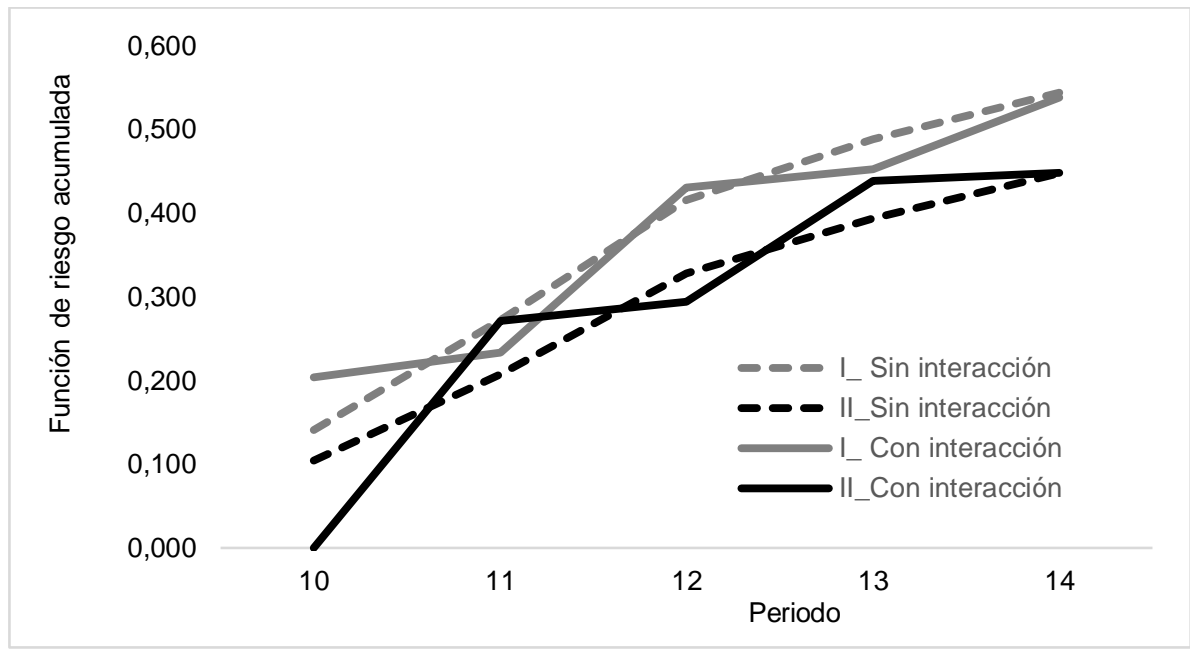

Fig. 7: Función de riesgo acumulada de graduación según semestre de ingreso con y sin interacción con el tiempo

Una vez evaluado el supuesto de proporcionalidad, se procedió a evaluar separadamente el efecto de cada una de las variables independientes en el riesgo de deserción y graduación. Posteriormente, se estimó el modelo completo con todas las variables. En la tabla 7 se presentan tanto las medidas crudas como ajustadas. Las medidas crudas muestran que a excepción del sexo, la edad y el semestre de ingreso, todas las variables presentaron asociación con la probabilidad de deserción (valor $p<0.05$ ). Así mismo, se encontró que todas las variables tuvieron un efecto significativo en la probabilidad de graduación (valor p < 0.05) (Tabla 7). En la Tabla 7, el perfil de línea base ( las 14 dummies del tiempo). se incluyeron en los modelos. pero los resultados no se presentan en la tabla. La variable semestre de ingreso no tiene información para el periodo 12 en deserción y para el periodo 10 en graduación debido a la poca cantidad de observaciones para estimar los coeficientes.

Las medidas ajustadas para ambos desenlaces muestran que: tener una edad de 18 años o más con respecto a tener menos de 18 años, reduce en un $47.1 \%$ la probabilidad de graduarse, comparado con persistir en la universidad cuando las demás variables permanecen constantes. Sin embargo, esto no influencia significativamente el riesgo de desertar. Provenir de un hogar con ingresos familiares entre 2 y 5 SMMLV con respecto a proceder de uno donde los ingresos son menores de 2 SMMLV, disminuye el riesgo de desertar con respecto a permanecer matriculado en la universidad en un $35.0 \%$, cuando las demás variables se mantienen constantes (Tabla 7)

Aquellos estudiantes, que estaban matriculados en un programa perteneciente a las ciencias de la salud o las ciencias sociales, comparado con estar matriculado en un programa de agronomía, veterinaria y afines, tuvieron 3.91 y 4.78 veces respectivamente, mayor probabilidad de graduarse con respecto a permanecer matriculado. Alcanzar un puntaje medio o alto en el examen de Estado para el ingreso a la educación superior, comparado con obtener un puntaje bajo, disminuye en $33.5 \%$ y $59.5 \%$ respectivamente, el riesgo de desertar con relación a permanecer matriculado. Por su parte, los estudiantes que obtuvieron un puntaje medio o alto en el examen de examen de Estado para el ingreso a la educación superior tuvieron 0.87 y 3.49 veces mayor probabilidad de graduarse que de permanecer matriculado (Tabla 7) 
Los estudiantes que a lo largo de su trayectoria académica experimentaron reprobación de al menos una asignatura, tuvieron 3.71 veces mayor riesgo de desertar que permanecer matriculado en la universidad. Así mismo, esta condición disminuyó la probabilidad de graduarse, comparado con persistir en la universidad en un $75.1 \%$. Con respecto al semestre en el que ingresa el estudiante, el término de interacción muestra que esta variable sólo tiene efecto para la graduación. Para los periodos 11 y 13 aquellos estudiantes que ingresaron en el segundo semestre tuvieron 10.4 y 5.1 veces respectivamente, mayor probabilidad de graduarse que de permanecer en la universidad. Por otra parte, para los periodos 12 y 14, ingresar en el segundo semestre del año, disminuyó la probabilidad de graduarse en un $89.5 \%$ y $90.3 \%$ respectivamente, comparado con permanecer matriculado en la universidad (Tabla 7).

\section{DISCUSIÓN}

La deserción se constituye en un fenómeno complejo ya que no existe una definición teórica y operativa única debido a las diversas ópticas desde las cuales se puede abordar (Tinto, 1989). En ese sentido, la OCDE, en su informe de indicadores hace alusión a la diversidad de factores institucionales y características específicas de los sistemas educativos de los diferentes países que pueden ayudar a explicar las variaciones en los diferentes indicadores, los cuales, a su vez, también pueden ser heterogéneos debido al tipo y fuente de datos que se utilicen para su medición. Adicionalmente, estas diferencias también pueden observarse al interior de los países, donde la especificidad de los contextos institucionales en los que se estudie la problemática conlleva a que los resultados obtenidos en las diversas investigaciones no siempre sean extrapolables. Aunado a lo anterior, la complejidad en la aproximación al fenómeno puede darse también desde la definición operativa de la deserción con respecto al número periodos académicos consecutivos sin vinculación a partir del cual un estudiante puede considerarse como desertor (Castro et al., 2020), hasta la determinación del tiempo de seguimiento mínimo requerido para la observación del fenómeno y el tipo de deserción en términos de retiro del programa, de la universidad o del sistema educativo (Heublein, 2014). Esto implica que la definición que se adopte en los diferentes estudios también deberá tenerse en consideración al momento de analizar y comparar las cifras observadas en diferentes contextos.

Con respecto a la definición del evento, en los análisis de supervivencia para deserción y graduación bajo modelos de riesgos competitivos reportados en la literatura, se ha encontrado que, algunos la han adoptado a nivel de programa académico (Carreira y Lopes, 2019; Castaño et al., 2006; Osorio et al., 2012), mientras que otros lo hicieron a nivel de universidad (Clerici et al., 2015; Ortiz y Dehon, 2013) o del sistema educativo (Reisel y Brekke, 2010). Para el presente estudio, sólo fue posible definirla a nivel de programa académico, debido a que no pudo obtenerse información acerca del destino del estudiante una vez desertaban. Esta situación es común en muchas instituciones educativas, debido a que los estudiantes no están obligados a notificar los motivos de su retiro, además, pueden existir restricciones normativas para acceder a la información de los estudiantes sin su previa autorización (Heublein, 2014).

Por otro lado, con relación al tiempo de seguimiento, se debe tener en consideración el tiempo adicional de observación con respecto a la duración teórica del programa, ya que un mayor tiempo de seguimiento permite observar una mayor tasa de finalización. En ese sentido, los indicadores de la OCDE para 2017 evidenciaron que, en promedio, estas tasas incrementaron 28 puntos porcentuales cuando se calcularon tres años después de la duración teórica del programa (OECD, 2019). En las investigaciones reportadas en la literatura sobre deserción y graduación bajo modelos de riesgos competitivos, se pudo identificar que algunos realizaron seguimiento por año y medio (Castaño et al., 2006; Osorio et al., 2012), otros por dos (Carreira y Lopes, 2019; Clerici et al., 2015), tres (Shea y Bidjerano, 2016) y hasta por ocho años más de la duración teórica del programa (Ortiz y Dehon, 2013). Si bien no existe consenso frente el número mínimo de periodos adicionales que debería considerarse para hacer seguimiento (Heublein, 2014), sugiere que, una regla para determinar el tiempo mínimo adicional de observación puede ser a partir de la determinación de la proporción de estudiantes que aún están matriculados en la institución en el último periodo, donde ésta no sea superior al $20 \%$ del total de los que iniciaron. En el presente estudio, se siguieron los estudiantes por dos años más de la duración teórica del programa; para éste el último periodo, la proporción de estudiantes fue del 16\%.

Con relación a la graduación, en la presente investigación se encontró que la probabilidad de graduarse en el tiempo fue de 0.187, cifra similar a la reportada por (Ortiz y Dehon, 2013) entre estudiantes de una universidad de bélgica donde fue de 0.178 . En ese mismo sentido, la presente investigación reportó una tasa de graduación hasta el periodo 14 del $49.0 \%$, la cual es similar al hallazgo encontrado por (Clerici et al., 2015) entre estudiantes de una universidad italiana que fueron seguidos por el mismo tiempo adicional donde la tasa fue del $47.0 \%$. Por su parte, (Ortiz y Dehon, 2013) encontraron una tasa de graduación del $43.6 \%$ entre estudiantes que fueron seguidos por 8 periodos adicionales. Si bien las investigaciones anteriormente descritas, se desarrollaron en países europeos donde las condiciones pueden ser diferentes al contexto latinoamericano, tienen la ventaja de que fueron desarrollados bajo el mismo modelo de riesgos competitivos utilizado en el presente estudio, el cual, como ya se mencionó en la sección introductoria, evita el problema de la sobreestimación de las probabilidades de ocurrencia de los eventos de interés. 


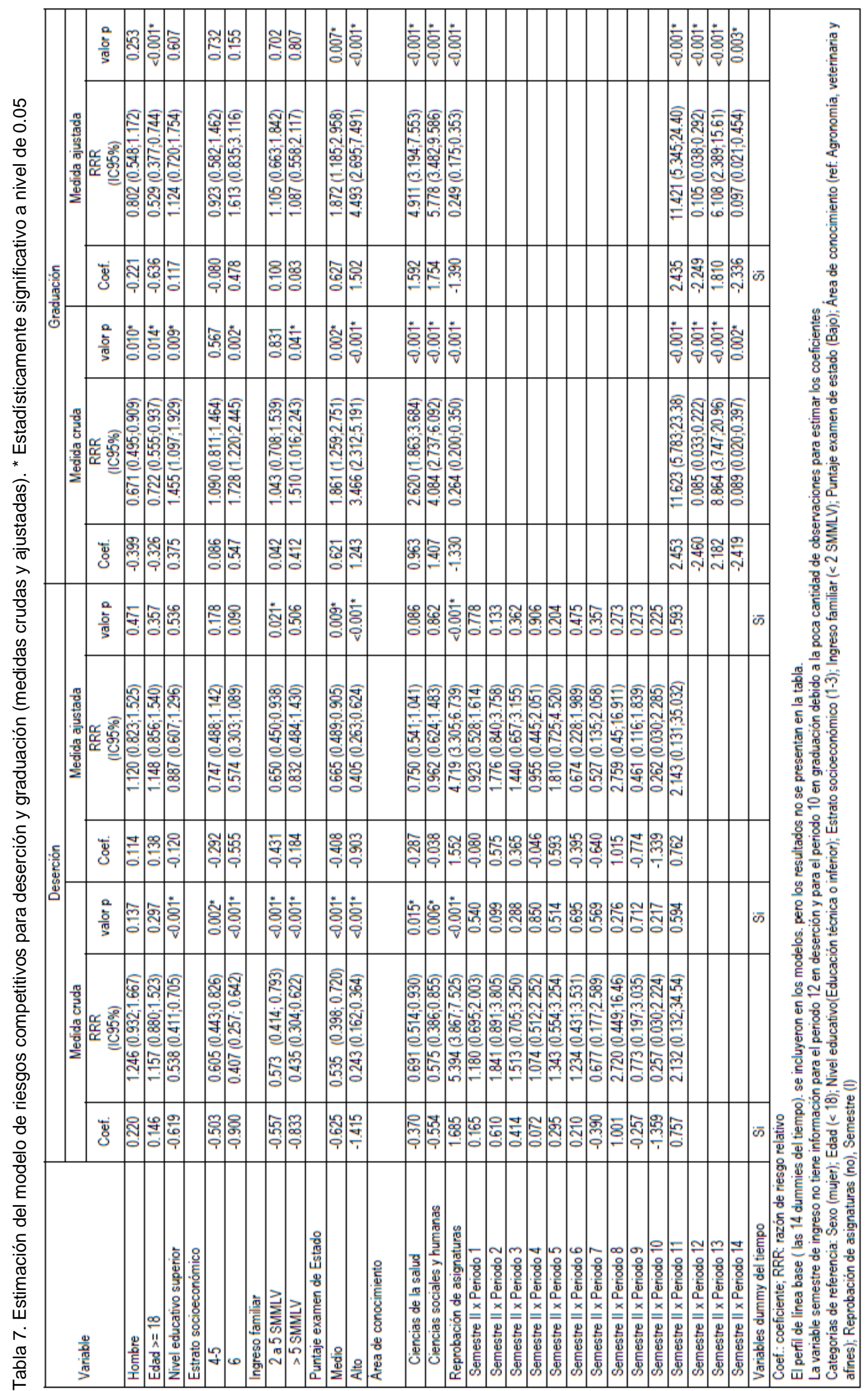


Con respecto a los factores asociados a la deserción y graduación, en el presente estudio no se encontró una asociación estadísticamente significativa con el sexo del estudiante, no obstante, aunque este mismo hallazgo fue encontrado por Osorio y Colaboradores (Osorio et al., 2012), otros estudios han mostrado que los hombres tienen significativamente mayor riesgo de desertar (Guerrero, 2018, Gartner et al., 2016) y menor probabilidad de graduarse (Carreira y Lopes, 2019; Ortiz y Dehon, 2013). Al analizar el efecto de las características socioeconómicas, a excepción de uno de los rangos de ingreso familiar (2 a 5 SMMLV), ninguna de las variables presentó un efecto estadísticamente significativo en la probabilidad de deserción o graduación. Frente a este hallazgo es importante tener en consideración que el presente estudio se llevó a cabo en una institución de educación superior de tamaño pequeño de carácter privado donde el $62 \%$ de los estudiantes tenían madres con estudios superiores, el $48 \%$ habitaban viviendas en estrato 4 o superior, el $35 \%$ provenían de hogares con ingresos superiores a los 5 SMMLV. Para el mismo periodo de estudio (2009-2010), en la población universitaria colombiana, estas cifras fueron de: 18\%, 10\%, 9\% (Ministerio de Educación Nacional, 2016). Los datos anteriormente descritos, sugieren que la población objeto de la presente investigación contaba con unas mejores condiciones socioeconómicas con respecto a la población universitaria general, lo que pudiera ayudar a explicar el efecto no significativo de estas características en el modelo. En este sentido, Castaño y Colaboradores (Castaño et al., 2006) encontraron hallazgos contradictorios entre distintos programas académicos con respecto al efecto del nivel educativo de la madre en la probabilidad graduación, donde se concluyen que las características de los programas y las universidades, sumadas a la educación de los padres, puede influir de manera importante en la permanencia del estudiante.

Frente a las condiciones académicas, tanto el puntaje en el examen de estado como la pérdida de asignaturas presentaron un efecto significativo en la probabilidad de deserción y graduación. Lo anterior refuerza la hipótesis de que tanto las condiciones académicas previas al ingreso como rendimiento académico influyen en la permanencia y graduación estudiantil. En ese sentido, algunos autores han encontrado que un mayor número de créditos reprobados influye negativamente en la probabilidad de graduación (Castaño et al., 2006) y que un menor promedio académico incrementa el riesgo de deserción (Osorio et al., 2012; Guerrero, 2018). Así mismo, se ha evidenciado que el promedio académico puede ser un buen predictor de deserción universitaria (Ramírez y Grandón, 2018). Por otro lado, con respecto a los exámenes de ingreso a la educación superior, otros estudios también han encontrado que puntajes más bajos se asocian con una mayor deserción (Guerrero, 2018; Carvajal et al., 2018; Miranda y Guzmán, 2017, Ramírez y Grandón, 2018). Este hallazgo, refuerza la hipótesis de que las condiciones en las que ingresa el estudiante, en términos de logros obtenidos durante su educación media, juegan un papel importante no sólo en el ingreso a una institución de educación superior de alta calidad sino en el desempeño académico a lo largo de la trayectoria educativa. En ese sentido,(Cuenca, 2016) afirma que los logros educativos y ocupacionales de los graduados de la educación superior colombiana están mediados por el origen social, en términos de los niveles socioeconómico y educativo de las familias, los cuales determinan el desempeño en la educación media, el cual a su vez, permite ingresar de manera más fácil e inmediata a una educación superior de mejor calidad.

Con respecto al ingreso de los estudiantes en los diferentes semestres académicos, la evidencia ha mostrado que éste tiende a estar relacionado con el periodo de finalización de la educación media. En Colombia, los estudiantes provenientes de colegios de calendario A (que empieza en el mes de febrero y culmina en el mes de noviembre) tienden ingresar a la universidad en el primer semestre, mientras que los de calendario $B$ (que inicia en el mes de septiembre y finaliza en el mes de junio) ingresan con mayor frecuencia en el segundo periodo. Para el año 2010 (Bonilla et al., 2014), las cifras de las inscripciones de los estudiantes de grado 11 para la presentación del examen de estado para el ingreso a la educación superior, mostraron que el $94 \%$ de todos los estudiantes y el $78 \%$ de los estudiantes de colegios no oficiales, presentaron sus pruebas en el mes de septiembre (usualmente asociado al calendario A).

En el presente estudio, se pudo evidenciar que el semestre en el que el estudiante ingresó a la universidad se asoció significativamente con la probabilidad de obtener el título. Para el periodo 14, la tasa de graduación entre quienes ingresaron en el primero periodo fue del $53.0 \%$, mientras que, en el otro grupo fue del $42.3 \%$. Adicionalmente, se observó que ésta no fue proporcional a lo largo del tiempo debido que ninguno de los estudiantes que ingresó en el segundo semestre logró titularse en el tiempo (comparado con un 18.5\% de los que ingresaron en el primer periodo). Para los periodos 11 y 13, fue mayor entre quienes ingresaron en el segundo semestre, mientras que, en los que ingresaron en el primero, las mayores tasas de graduación se observaron para los periodos 10, 12 y 14. En la presente investigación, no fue posible obtener el dato del calendario al que pertenecía el colegio de donde egresó el estudiante, sin embargo, la mayor proporción observada de estudiantes matriculados en el semestre I, sumado al comportamiento descrito en la sección anterior sobre las inscripciones de los estudiantes a los exámenes de estado, permite sugerir que gran parte de los estudiantes podrían provenir de colegios de calendario A; situación que pudiera explicar el hecho de que los estudiantes matriculados en el primer semestre, tuvieran un mejor desempeño en la educación media y por tanto un mejor rendimiento académico que les permitiera graduarse más tempranamente, contrario a los del segundo semestre, que posiblemente se corresponde con estudiantes que por su menor desempeño en las pruebas de estado no lograron ingresar de manera inmediata a la universidad. En estos estudiantes, 
puede ser bastante útil, fomentar estrategias de acompañamiento académico desde el primer semestre, de modo que logren fortalecer sus competencias para evitar rezagos en su trayectoria. Finalmente, el área de conocimiento sólo mostró un efecto significativo en la probabilidad de graduación. Este hallazgo difiere de los reportado por otros estudios donde encontraron que pertenecer a programas de ciencias de la salud o ciencias sociales no estaba relacionado con una mayor probabilidad de graduación (Ortiz y Dehon, 2013) pero sí con un mayor riesgo de deserción (Carvajal et al., 2018; Guerrero,2018). Las estadísticas a nivel nacional muestran un mayor riesgo de deserción entre programas pertenecientes a agronomía, veterinaria y afines (Ministerio de Educación Nacional, 2016), sin embargo, la relación de las funciones de deserción y graduación encontradas, mostraron que para el periodo 14, uno de cada cuatro estudiantes aún permanecía en la universidad sin graduarse pero tampoco sin desertar. Este rezago, puede estar relacionado por pérdidas de asignaturas durante la trayectoria académica o con retrasos en el cumplimiento de requisitos como trabajo de grado o segundo idioma (aspectos no incluidos en este estudio), los cuales no afectan la probabilidad de desertar pero que sí pueden retrasar el proceso de titulación.

Si bien el presente estudio se desarrolló bajo el modelo de tiempo discreto análisis de supervivencia en tiempo discreto para riesgos competitivos, el cual es el más adecuado para el abordaje del fenómeno en cuestión, y aunque se incluyó una variable variante en el tiempo relacionada con el rendimiento académico de los estudiantes, no fue posible incluir otras variables institucionales como, por ejemplo, con apoyos financieros o académicos, los cuales podría ayudar a una mejor comprensión del fenómeno. Para este tipo de estudios longitudinales, donde es fundamental contar con información veraz y completa de manera periódica, se sugiere que las instituciones de educación superior, además de fortalecer sus sistemas de información, también fomenten la cultura de la importancia de la información para la gestión y toma de decisiones.

\section{CONCLUSIONES}

Los resultados anteriores permiten concluir: 1) Si bien el mayor riesgo de deserción se observa en el primer semestre, 35 de cada 100 estudiantes que desertan, lo hace en los periodos cuatro a siete; 2) Si bien cerca de la mitad de los estudiantes se gradúa durante el tiempo de seguimiento de 14 periodos, sólo el $25.2 \%$ obtiene su título en el tiempo teórico del programa y el $24.9 \%$ tarda más de un año después del tiempo previsto por el programa; 3) Para el periodo 11, el $58.5 \%$ de los estudiantes ya había experimentado uno de los dos desenlaces, en su mayoría deserción, ya que las graduaciones sólo se comenzaron a observar a partir del periodo 10. Así mismo, para el periodo 14, aproximadamente dos de cada diez estudiantes aún permanecían en la universidad sin graduarse y sin desertar; 4) Las medidas ajustadas por el modelo evidenciaron que tener un ingreso familiar < 2 SMMLV, obtener un puntaje bajo en el examen de Estado para el ingreso a la educación superior y perder al menos una asignatura en el periodo incrementa la probabilidad de deserción. Por su parte, ingresar a la universidad con una edad inferior a 18 años, obtener un puntaje medio o alto en el examen de Estado para el ingreso a la educación superior, aprobar las asignaturas en cada periodo y cursar un programa perteneciente a las ciencias de la salud o las ciencias sociales incrementa la probabilidad de graduación.

\section{REFERENCIAS}

Aljohani, O., A, Comprehensive Review of the Major Studies and Theoretical Models of Student Retention in Higher Education, ISSN: 1925-475X, High. Educ. Stud., 6(2), 1-18 (2016a)

Aljohani, O., A Review of the Contemporary International Literature on Student Retention in Higher Education, ISSN: 22029478, IJELS, 4(1), 40-52 (2016b)

Bonilla, L., Sánchez, A., Barón, J., y otros cuatro autores, Educación y Desarrollo Regional en Colombia. Libros Banco de la República, Bogotá (2014)

Carreira, P., y Lopes, A., Drivers of Academic Pathways in Higher Education: Traditional vs. Non-Traditional Students, doi: 10.1080/03075079.2019.1675621, Stud. High. Educ., 1-16 (2019)

Carvajal, C., González, J., y Sarzoza, S., Variables Sociodemográficas y Académicas Explicativas de la Deserción de Estudiantes en la Facultad de Ciencias Naturales de la Universidad de Playa Ancha (Chile), doi: 10.4067/S071850062018000200003, Form. Univ., 11(2), 3-12 (2018)

Castaño, E., Gallón, S., Gómez, K., y Vásquez, J., Análisis de los Factores Asociados a la Deserción y Graduación Estudiantil Universitaria, ISSN: 0120-2596, Lect. Econ, (65), 11-35 (2006)

Castro, B., Manrique, R., González, D., y Segura, A., Trayectoria Académica y Factores Asociados a Graduación, Deserción y Rezago en Estudiantes de Programas de Pregrado de una Universidad Privada de Medellín (Colombia), doi: 10.4067/S0718-50062020000100043, Form. Univ., 13(1), 43-54 (2020)

CEDE. Universidad de los Andes. Investigación Sobre Deserción en las Instituciones de Educación Superior en Colombia. 2007.

Clerici, R., Giraldo, A., y Meggiolaro, S., The Determinants of Academic Outcomes in a Competing Risks Approach: Evidence From Italy, doi: 10.1080/03075079.2013.878835, Stud. High. Educ., 40(9), 1535-1549 (2015)

Cuenca, A., Desigualdad de Oportunidades en Colombia: Impacto del Origen Social Sobre el Desempeño Académico y los Ingresos de Graduados Universitarios, doi: 10.4067/S0718-07052016000200005, Estudios pedagógicos (Valdivia), 42(2), 69-93 (2016) 
DesJardins, S., Ahlburg, D., y McCall, B., A Temporal Investigation of Factors Related to Timely Degree Completion, doi: 10.1353/jhe.2002.0042, J. High. Educ, 73(5), 555-581 (2002)

Fishbein, M, y Ajzen, I., Belief, Attitude, Intention, and Behavior: An Introduction to Theory and Research, Reading, MA:Addison-Wesley (1975)

Guerrero, S., Caracterización de la Deserción en la Universidad Pedagógica y Tecnológica de Colombia Durante el Periodo 2008-2015, doi: 10.22507/rli.v15n1a2, Revista Lasallista de Investigación, 15(1),16-28 (2018)

Gartner, M., Dussán, C., y Montoya, D., Caracterización de la Deserción Estudiantil en la Universidad de Caldas en el Período 2009-2013. Análisis a partir del Sistema para la Prevención de la Deserción de la Educación Superior-SPADIESdoi: 10.22507/rli.v15n1a2 , Revista Latinoamericana de Estudios Educativos (Colombia), 12(1), 132-158 (2016)

Heublein, U., Student Drop-out from German Higher Education Institutions, doi: 10.1111/ejed.12097, Eur. J. Educ., 49(4), 497-513 (2014)

Meyer, B., Unemployment Insurance and Unemployment Spells, doi: 10.2307/2938349, Econometrica, 58(4), 757-82 (1990)

Miranda, M., y Guzmán, J., Análisis de la Deserción de Estudiantes Universitarios Usando Técnicas de Minería de Datos, doi: 10.4067/S0718-50062017000300007, Form. Univ.,10(3), 61-68 (2017)

Ministerio de Educación Nacional, Compendio Estadístico de la Educación Superior Colombiana. Imprenta Nacional de Colombia, Bogotá (2016)

OECD, How Many Students Complete Tertiary Education?, in Education at a Glance 2019: OECD Indicators, 208-227, OECD Publishing, Paris (2019)

Ortiz, E., y C. Dehon, Roads to Success in the Belgian French Community's Higher Education System: Predictors of Dropout and Degree Completion at the Université Libre de Bruxelles, doi: 10.1007/s11162-013-9290-y, Research in Higher Education (RHEJ), 54(6), 693-723 (2013)

Osorio, A., Bolancé, C. y Castillo, M., Deserción y Graduación Estudiantil Universitaria: una Aplicación de los Modelos de Supervivencia, doi: 10.22201/iisue.20072872e.2012.6.55, Rev. Iberoam. Educ. Super, III(6), 31-57 (2012)

Prentice, R. y Gloeckler, L., Regression Analysis of Grouped Survival Data with Application to Breast Cancer Data, doi: 10.2307/2529588, 34(1), 57-67 (1978)

Ramírez, P., y Grandón, E., Predicción de la Deserción Académica en una Universidad Pública Chilena a través de la Clasificación basada en Árboles de Decisión con Parámetros Optimizados, doi: 10.4067/S0718-50062018000300003, Form. Univ., 11(3), 3-10 (2018)

Reisel, L. y Brekke, I., Minority Dropout in Higher Education: A Comparison of the United States and Norway Using Competing Risk Event History Analysis, ISSN: 0266-7215, Eur. Sociol. Rev., 26(6), 691-712 (2010)

Scott, A. y Kennedy, B., Pitfalls in Pathways: Some Perspectives on Competing Risks Event History Analysis in Education Research, doi: 10.3102/10769986030004413, J. Educ. Behav. Stat., 30(4), 413-442 (2005)

Shea, P. y Bidjerano, T., A National Study of Differences between Online and Classroom-Only Community College Students in Time to First Associate Degree Attainment, Transfer, and Dropout, ISSN 1939-5256, Online Learning, 20(3), 14-15 (2016)

Singer, J. y Willett, J., It's About Time: Using Discrete-Time Survival Analysis to Study Duration and the Timing of Events, doi: 10.3102/10769986018002155, J. Educ. Behav. Stat.,18(2), 155-195 (1993)

Spady, W., Dropouts from Higher Education: Toward an Empirical Model, doi: 10.1007/BF02282469, Interchange, 2(3), 38-62 (1971)

Tinto, V., Dropout from Higher Education: A Theoretical Synthesis of Recent Research, doi: 10.3102/00346543045001089, Review of Educational Research, 45(1), 89-125 (1975)

Tinto, V., Definir la Deserción una Cuestión de Perspectiva, ISSN: 0185-2760, Rev. Educ. Sup, 3(71), 33-51 (1989)

Tinto, V., Leaving College: Rethinking the Causes and Cures of Student Attrition. 2a Ed. Chicago, IL: University of Chicago Press (1993)

Tinto, V., Research and Practice of Student Retention: What Next?, doi: 10.2190/4YNU-4TMB-22DJ-AN4W, J Coll Stud Ret, 8(1), 1-19 (2006)

Tinto, V., From Theory to Action: Exploring the Institutional Conditions for Student Retention. In: Smart J. (eds) Higher Education: Handbook of Theory and Research. Higher Education: Handbook of Theory and Research, vol 25. Springer, Dordrecht (2010)

Urrego, M., La Investigación Sobre Deserción Universitaria en Colombia 2006-2016: Tendencias y Resultados. Pedagogía y saberes, doi: 10.17227/pys.num51-8664, 51, 49-66 (2019)

Van der Haert, M., Ortiz, E., y otros tres autores, Are Dropout and Degree Completion in Doctoral Study Significantly Dependent on Type of Financial Support and Field of Research?, doi: 10.1080/03075079.2013.806458, Stud. High. Educ., 39(10), 1885-1909 (2014)

Zewotir, T., North, D. y Murray, M., The time to Degree or Dropout Amongst Full-Time Master's Students at University of KwaZulu-Natal, doi: 10.17159/SAJS.2015/20140298, S. Afr. J. Sci., 111(9-10), 01-06 (2015) 